\title{
comportement des tirants d'ancrage dans un sable fin
}

\author{
par \\ A.G. Davis \\ Chef de la Division Mécanique des Sols et des Chaussées au C.E.B.T.P. \\ c. Plumelle \\ Chercheur au C.E.B.T.P. \\ Chef de Travaux au C.N.A.M.
}

Conférence présentée devant le Comité Français de mécanique des sols et fondations le 18 juin 1979

\section{But de la recherche}

Le développement très important que connait actuellement la technique des tirants d'ancrage a entraîné la réalisation d'essai in situ en vraie grandeur parmi lesquels on peut citer les essais réalisés en France par Bustamante, Delmas et Lacour [1] et en Allemagne par Ostermayer et Scheele [2]. Ce type d'essais met en jeu un grand nombre de paramètres : hétérogénéité du sol, pression d'injection, quantité de coulis injectée, forme irrégulière du bulbe, procédé de mise en œuvre, etc. Le nombre de ces facteurs et la relative indétermination de la connaissance de certains d'entre eux compliquent l'interprétation des résultats obtenus.

Afin d'améliorer la précision de la connaissance des paramètres mis en jeu et d'en réduire le nombre, les essais qui font l'objet de cet article ont porté sur des tirants d'ancrage préfabriqués dont le bulbe était constitué par un cylindre métallique ou un cylindre en coulis de ciment, de diamètre constant et de longueur variable et qui étaient ancrés horizontalement, à des profondeurs variables, dans un massif de sable compacté à une densité constante.

Les études ont porté sur :

- La capacité d'ancrage en fonction de la longueur de bulbe et en fonction de la hauteur du sable de recouvrement,

- La détermination des courbes de fluage et de la traction critique du fluage suivant les Recommandations TA 77 .

- Les déplacements de la tête et du pied de l'ancrage en fonction des charges appliquées.

- Les études des contraintes développées dans le sol devant la tête de l'ancrage, et autour du corps de l'ancrage,

- La répartition de l'effort dans le tirant d'ancrage en fonction des charges appliquées,

- La distribution du frottement latéral unitaire en fonction des charges appliquées et du déplacement local du tirant d'ancrage par rapport au sol,

- Les délimitations des zones de ruptures du sol devant la tête de l'ancrage et autour du corps d'ancrage.

\section{Dispositif d'essai}

Le dispositif est schématisé sur la figure 1. Les efforts appli- qués au tirant étaient repris par un mur de soutènement dont les contreforts étaient renforcés par un chevêtre composé de deux HEB munis en leur milieu d'une plaque de répartition.

\section{Caractéristiques du sable utilisé}

Le sable utilisé, de granulométrie uniforme, était du type sable de Fontainebleau.

Les densités minimum et maximum étaient respectivement égales à 1,31 et 1,69. La densité adoptée était égale à 1,51. Cette valeur correspond à un sable moyennement dense $\left(I_{D}=0,59\right)$ et de densité supérieure à la densité critique. L'angle de frottement correspondant à cette densité, et mesuré à la boite de cisaillement était $\varphi^{\prime}=33^{\circ}$, compte tenu de la correction de dilatance de Bishop.

Les valeurs de l'angle de frottement sable sur acier sablé étaient : $\varphi \max =30^{\circ}$ et $\varphi \min =28^{\circ}$.

\section{Programme d'essais}

Le programme d'essais est illustré par la figure 2. Il comportait :

- Essai $n^{\circ} 1$ : plaque circulaire, avec une hauteur de sable $\mathrm{h}_{\mathrm{s}}=2 \mathrm{~m}$

- Essail $n^{\circ} 2$ : tirant métallique, de $2 \mathrm{~m}$ en traction, avec $\mathrm{h}_{\mathrm{s}}=2 \mathrm{~m}$

- Essai $n^{\circ} 3$ : tirant métallique de $2 \mathrm{~m}$ en compression, avec $\mathrm{h}_{\mathrm{s}}=2 \mathrm{~m}$

- Essai no 4 : tirant en coulis de ciment de $2 \mathrm{~m}$ avec $h_{\mathrm{s}}=2 \mathrm{~m}$

- Essai $n^{\circ} 5$ : tirant en coulis de ciment de $3 \mathrm{~m}$ avec $\mathrm{h}_{\mathrm{s}}=2 \mathrm{~m}$

- Essai $n^{\circ} 6$; tirant en coulis de ciment de $4 \mathrm{~m}$ avec $\mathrm{h}_{\mathrm{s}}=2 \mathrm{~m}$

- Essai $n^{\circ} 7$ et 8 : tirant en coulis de ciment de $3 \mathrm{~m}$ avec $\mathrm{h}_{\mathrm{s}}=3 \mathrm{~m}$

- Essai $n^{\circ} 9$ : tirant en coulis de ciment de $3 \mathrm{~m}$ manchonné avec $h_{\mathrm{s}}=3 \mathrm{~m}$.

Les tirants $n^{\circ} 7$ et 8 avaient des caractéristiques identiques, dans le but d'apprécier la dispersion des résultats. 


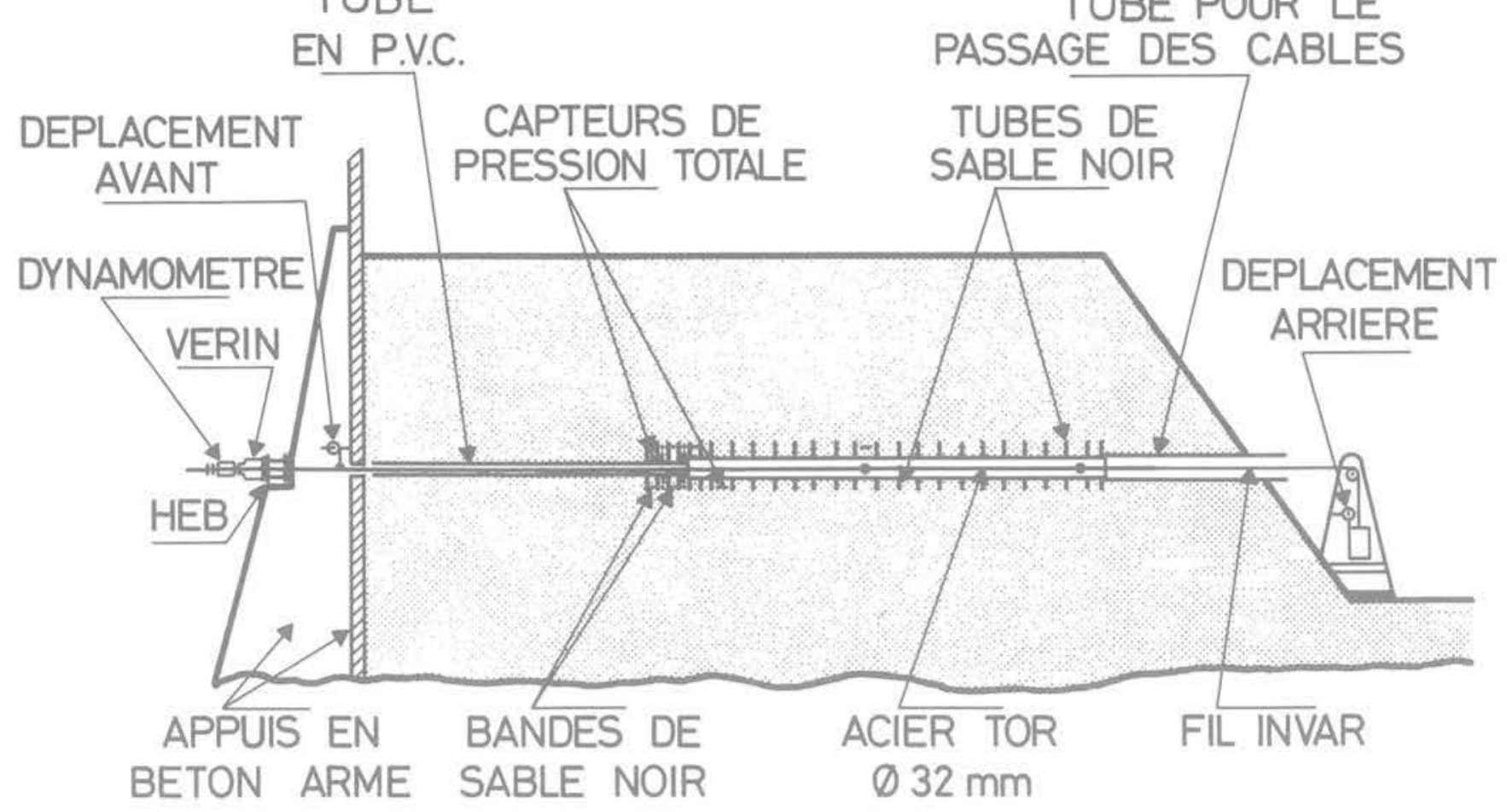

Fig. 1 Schéma du dispositif d'essai

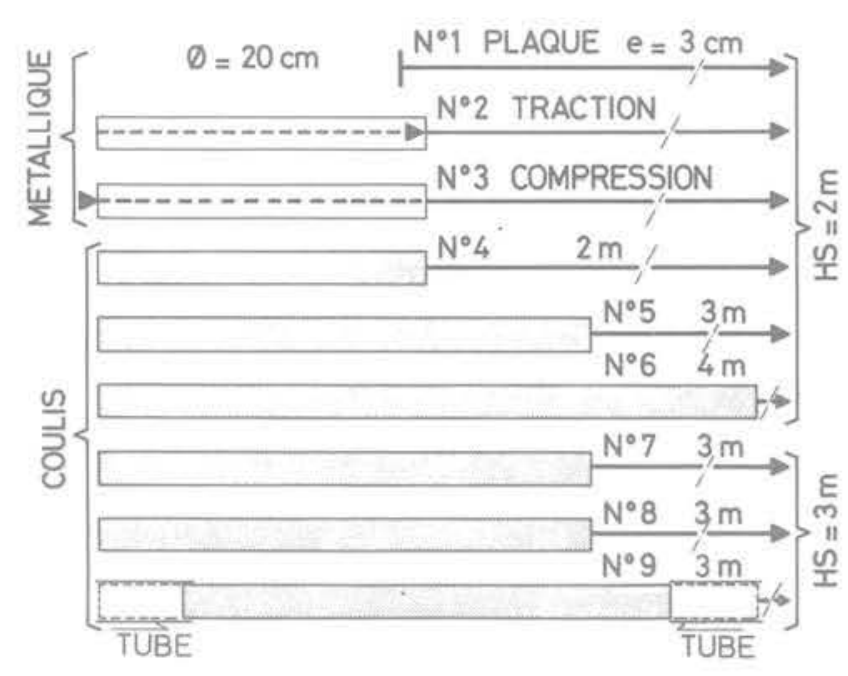

Fig. 2 Programme d'essai

\subsection{Tirants métalliques}

Les tirants d'ancrage métalliques ( $n^{\circ} 2$ et 3 ) étaient constitués par deux demi-tubes de $2 \mathrm{~m}$ de longueur et de $194 \mathrm{~mm}$ de diamètre extérieur et $188 \mathrm{~mm}$ de diamètre intérieur. Des jauges de déformation ont été placées à l'intérieur des demi-tubes, avant leur assemblage par points de soudure. Les extrémités ont été obturées par deux plaques circulaires boulonnées.

Une tige en acier de $30 \mathrm{~mm}$ de diamètre a été boulonnée en tête du tirant essayé en traction et en pied du tirant essayé en compression.

La surface des cylindres a été enduite de colle et sablée avec le sable utilisé pour les essais.

\subsection{Tirants en coulis de ciment}

Le bulbe de ces tirants était constitué par un cylindre de 200 $\mathrm{mm}$ de diamétre en coulis de ciment (avec un dosage ciment sur eau égal à 2) coulé dans des moules en PVC. L'armature était constituée par un acier T de $32 \mathrm{~mm}$ de diamètre.

Des jauges de déformation, à trame pelliculaire, ont été collées sur l'armature. Des jauges de $60 \mathrm{~mm}$ de longueur et enrobées de résine ont été placées dans le coulis, pendant le coulage. La surface des cylindres a également été sablée. Ces tirants ont été conservés sous une couche de sable humide pendant un mois avant essai.

\section{Dispositifs d'essai} extrémités lear un tube creux de diamètre à chacune de ses rieur au bulbe en vue d'éliminer l'effet de butée frontale.

\subsection{Tirant constitué par une plaque circulaire}

L'ancrage était constitué par une plaque circulaire épaisse en acier, de $200 \mathrm{~mm}$ de diamètre, boulonnée sur une tige en acier de $30 \mathrm{~mm}$ de diamètre.

Cet essai avait pour but de déterminer la butée devant le tirant.

\subsection{Dispositif de mise en traction des tirants (fig. 2)}

La tige de $30 \mathrm{~mm}$ des tirants métalliques ou l'armature en acier TOR des tirants en coulis était assemblée à une tige d'acier à haute résistance de $30 \mathrm{~mm}$ de diamètre. Dans sa partie située dans le massif de sable cette dernière tige se déplaçait dans une gaine en PVC. Elle traversait le mur de soutènement, passait entre les deux HEB du chevêtre et 


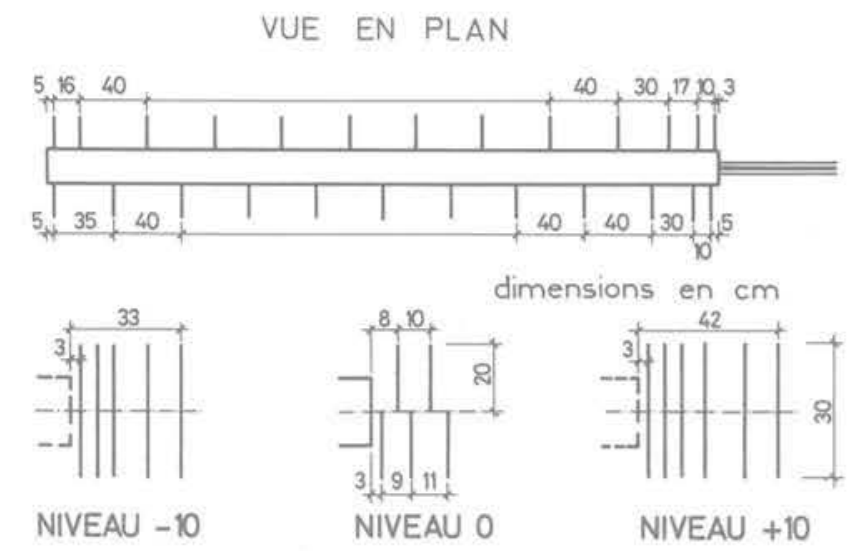

Fig. 3 Disposition des bandes de sable noir

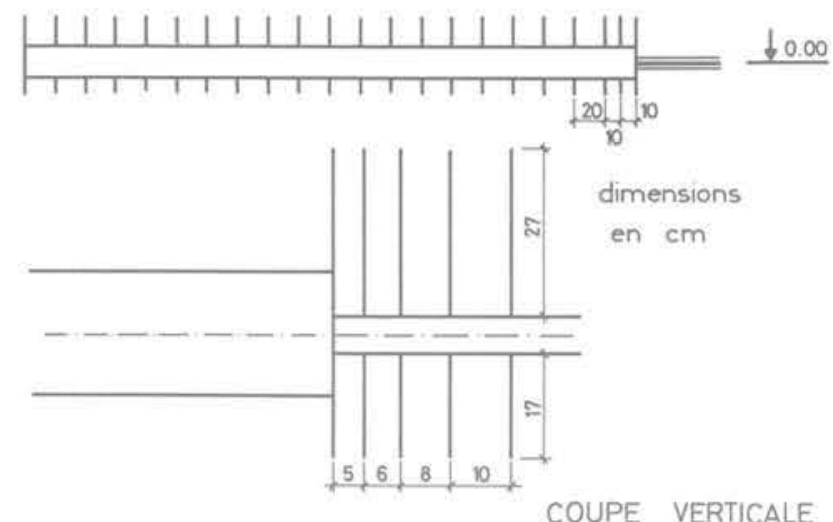

Fig. 4 Disposition des tubes de sable noir était boulonnée derrière le vérin et le dynamomètre. Les charges ont été maintenues constantes à $100 \mathrm{~N}$ près. Les déplacements de la tige, pendant l'essai, ont été mesurés au moyen d'un comparateur gradué en centièmes de $\mathrm{mm}$ et fixé sur la tige à la sortie de la traversée du mur de soutènement.

Derrière le bulbe du tirant et dans son axe était placé un tube en amiante ciment qui débouchait à travers le talus du massif de sable. Ce tube permettait d'une part la sortie des câbles reliés aux jauges de déformation et d'autre part de fixer un fil invar à l'extrémité arrière de l'armature du tirant et dans son axe. Ce fil après passage sur une poulie était tendu par un contre-poids dont le déplacement, mesuré par un comparateur gradué en centièmes de $\mathrm{mm}$, indiquait le déplacement du pied du bulbe du tirant.

\subsection{Mesure des déplacements du milieu sableux autour du tirant}

Les déplacements du milieu sableux à la fin de l'esai, ont été déterminés au moyen de bandes horizontales et de tubes verticaux constitués en sable noir.

Des bandes horizontales en sable noir ont été disposées devant la tête de l'ancrage à la cote 0 (niveau de l'axe du tirant) à la cote plus $10 \mathrm{~cm}$ et à la cote moins $10 \mathrm{~cm}$, ainsi que de part et d'autre de l'ancrage à la cote 0 (fig. 3 ).

Les tubes verticaux en sable noir ont été placés devant la tête de l'ancrage, au-dessus du corps d'ancrage et dans son axe et au-dessous du corps d'ancrage et dans son axe (fig. 4).

\subsection{Mesure des pressions transmises au milieu sableux}

L'évolution de ces pressions, durant les essais, a été mesurée au moyen de capteurs de pression totale placés devant la tête, en contact avec elle et autour du corps de l'ancrage à une distance de l'ordre de $5 \mathrm{~cm}$. Les emplacements de ces capteurs pour deux des tirants sont indiqués sur les figures 11,12 , et 13 . L'un de ces capteurs est visible sur la photographie de la figure 26.

\subsection{Mesure des efforts dans le tirant}

Ces efforts ont été mesurés au moyen de jauges de déformation, à trame pelliculaire, collées à l'intérieur des demi tubes des tirants métalliques ou sur l'armature des tirants en coulis de ciment.

Les efforts à l'intérieur du coulis ont été mesurés au moyen de jauges à béton placées dans ce coulis.

\section{Déroulement d'un essai}

\subsection{Etalonnage des tirants}

Avant essai les tirants métalliques ont été étalonnés en traction (essai 2) et en compression (essai 3) par application de trois cycles de chargement. Après leur période de conservation d'un mois sous une couche de sable humide, les tirants en coulis ont été étalonnés en traction, sous une charge inférieure à celle entraînant la fissuration. Ces étalonnages ont permis de connaître directement la réponse des jauges en fonction de la charge à laquelle elles étaient soumises.

\subsection{Mise en place du sable}

Le sable a été compacté par couches de $20 \mathrm{~cm}$ jusqu'à la cote moins $10 \mathrm{~cm}$, par couche de $10 \mathrm{~cm}$ entre les cotes moins $10 \mathrm{~cm}$ et plus $10 \mathrm{~cm}$ et par couches de $20 \mathrm{~cm}$ audessus de la cote plus $10 \mathrm{~cm}$. A chaque niveau les densités ont été contrôlées par mesure de densité au sable.

\subsection{Essai des tirants}

La traction de rupture $T$ a été fixée a priori. Le tirant a ensuite été soumis à des paliers successifs de chargement et croissant par valeurs égales au dixième de la charge de rupture supposé. Chaque palier a été maintenu pendant soixante minutes afin d'obtenir les courbes de fluage.

Les déplacements de la tête et du pied du bulbe d'ancrage ont été mesurés.

Pour chaque palier de chargement les courbes de fluage ont été tracées, donnant l'évolution des déplacements cumulés en fonction de log $t$, pendant les soixante minutes de chargement. Le phénomène de fluage a été caractérisé par la pente des droites de fluage ou de la tangente à la fin de la courbe de fluage. La courbe donnant l'évolution des pentes en fonction des valeurs des paliers de chargement permet de définir la traction critique $T_{C}$ (fig. 5). L'essai a été arrêté généralement après deux ou trois paliers de chargement de valeur supérieure à $T_{C}$.

Les mesures des jauges de déformation ont été réalisées au début et à la fin de chaque palier. Les mesures des capteurs de pression totale ont été enregistrées en continu pendant toute la durée de l'essai.

\subsection{Observation après essai}

Le déplacement du milieu sableux à la fin de l'essai a été déterminé par l'observation des déformations des bandes et des tubes en sable noir. 


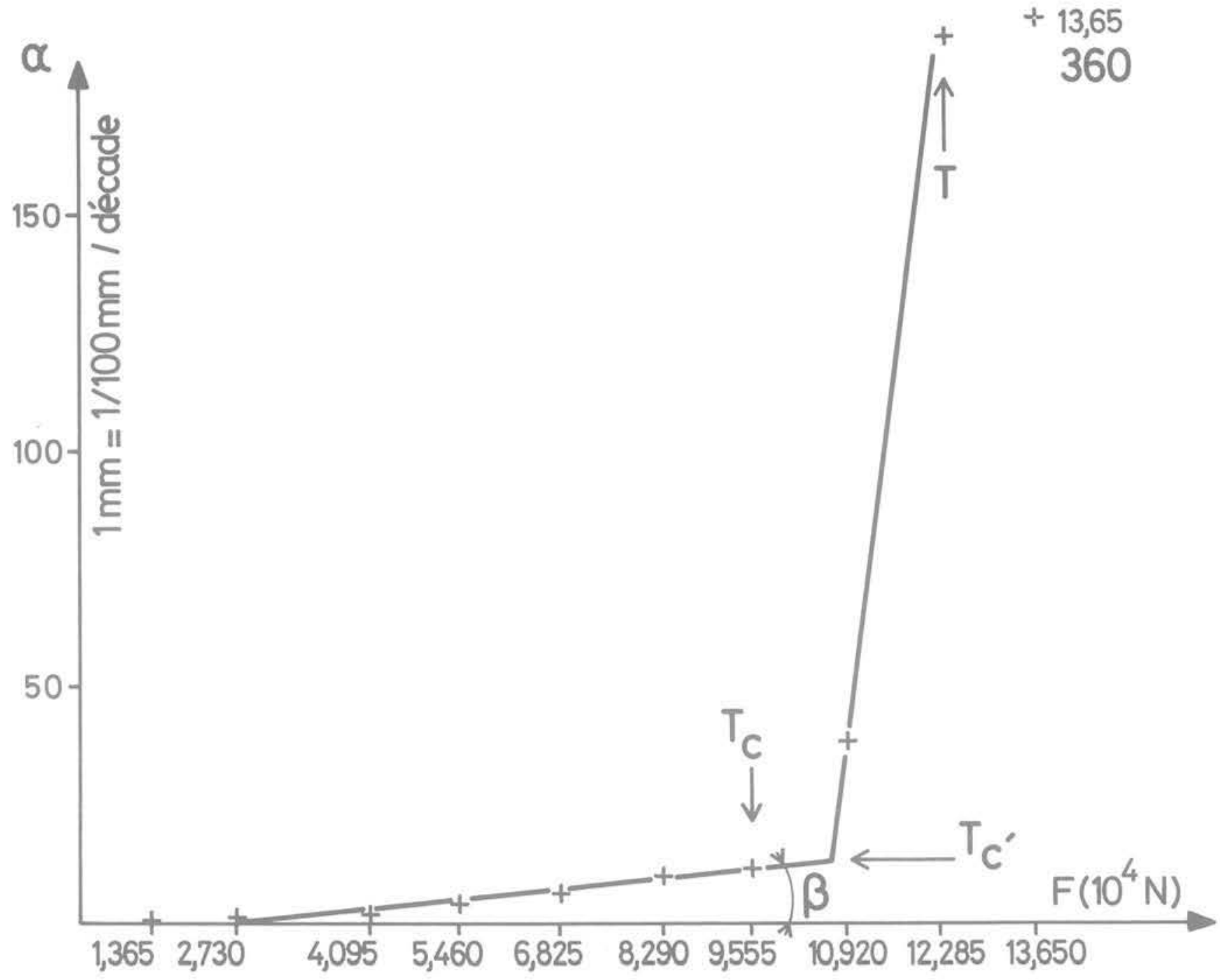

Fig. 5 Evolution des pentes $\alpha$ en fonction des tractions appliquées au tirant et définition des paramètres $T_{C}, T_{C}^{\prime}$ et $T$

Les fissures des ancrages en coulis ont également été relevées.

\section{Résultats}

\subsection{Traction critique $T_{C}$}

On a adopté comme valeur de $T_{C}$ la charge correspondant au dernier palier de chargement de la partie de faible pente $\beta$ de la courbe donnant la pente $\alpha$ en fonction de la charge (fig. 5).

La charge $T^{\prime}$ c correspond à l'intersection des deux droites schématisant cette courbe. La charge T correspond au premier palier présentant une valeur importante de la pente $\alpha$, de l'ordre de $1 \mathrm{~mm}$ par décade de minutes. Cet accroissement de pente se produit généralement au deuxième palier de chargement supérieur à $T_{C}$.

Les évolutions pour tous les tirants des pentes $\alpha$, en fonction des tractions appliquées au tirant ont été regroupées sur la figure 6. A titre indicatif, a également été reportée la courbe correspondant au tirant constitué par une plaque.
Les valeurs numériques des pentes $\beta$ de la partie de la courbe passant par l'origine, de $T_{C}$. $T_{C}$ ' et $T$ sont indiquées dans le tableau $n^{\circ} 1$. On peut constater que les tirants métalliques ( $n^{\circ} 2$ et 3 ) essayés respectivement en traction et en compression et le tirant $n^{\circ} 4$ en coulis dont les longueurs de bulbe sont identiques $(2 \mathrm{~m})$ ont des valeurs de $T_{C}$ groupées autour de $50 \mathrm{kN}$ (respectivement 49,2 - 56,4 et $57,3 \mathrm{kN}$ ).

Les tirants $n^{\circ} 7$ et 8 de longueur de bulbe identique $(3 \mathrm{~m})$ ont des valeurs de $T_{C}$ très voisines (respectivement 90,1 et $95,6 \mathrm{kN}$ ). Par contre le tirant $n^{\circ} 9$ de même longueur de bulbe que les précédents, mais dont la butée en tête avait été supprimée, a une valeur de $T_{C}(65,5 \mathrm{kN})$ de l'ordre de $30 \%$ inférieure à celle de ces tirants.

Le tirant $n^{\circ} 5$ de $3 \mathrm{~m}$ de longueur de bulbe et placé sous une hauteur de sable de 2 mètres à une valeur de $T_{C}(96,1 \mathrm{kN})$ semblable à celles correspondantes aux tirants $n^{\circ} 7$ et 8 (respectivement 90,1 et $95,6 \mathrm{kN}$ ) de même longueur de bulbe mais placés sous une hauteur de sable de $3 \mathrm{~m}$. Ces résultats montrent que la profondeur critique, au-delà de laquelle les efforts sont indépendants de la profondeur d'ancrage, est inférieure à $2 \mathrm{~m}$.

Les valeurs de $T_{C}$ obtenues pour les tirants n 4,5 et 6 (respectivement $57,3 \mathrm{kN}-96,1 \mathrm{kN}$ et $122,9 \mathrm{kN}$ ) de 2,3 et $4 \mathrm{~m}$ de longueur de bulbe montrent que pratiquement $T_{C}$ croit linéairement avec la longueur du bulbe (fig. 7). 


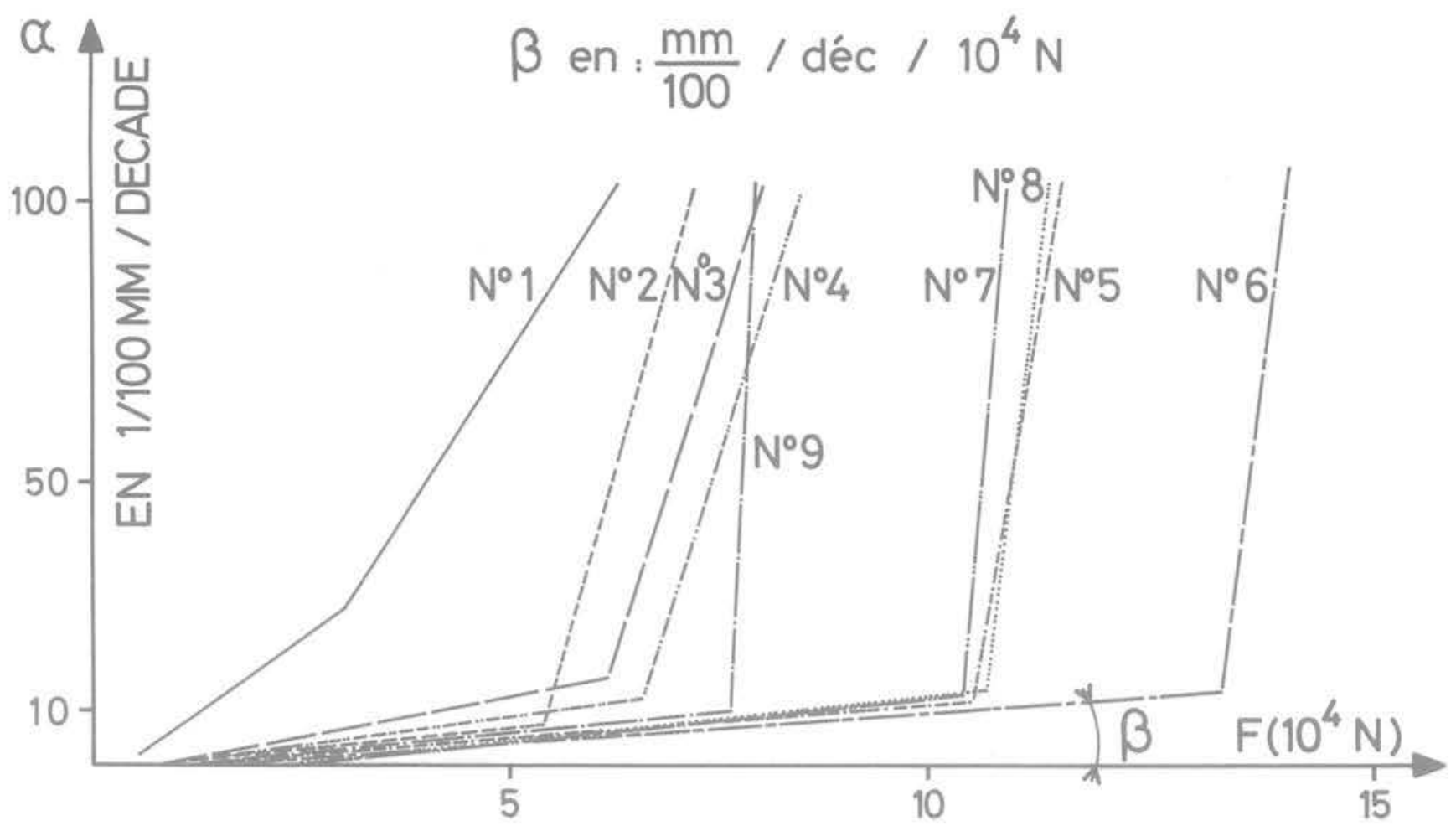

Fig. 6 Evolution des pentes $\alpha$ en fonction des tractions appliquées aux tirants

Les valeurs du rapport $\frac{T_{c}}{T_{c}}$ sont indiquées dans le tableau n०1. Les valeurs obtenues sont voisines de la valeur 0,9 proposée dans le document "Recommandations TA 77".

Dans le tableau $n^{\circ} 1$ ont également été reportées les valeurs de la traction admissible $T$ définies suivant les trois critères suivants :

$\mathrm{T}_{\mathrm{a}}=0,9 \mathrm{~T}_{\mathrm{c}}$

$T_{\mathrm{a}}=0,8 \mathrm{~T}_{\mathrm{c}}^{\mathrm{c}}$

$\mathrm{T}_{\mathrm{a}}=\frac{\mathrm{T}}{1,5}$

On peut constater que, sauf pour le tirant $n^{\circ} 2$, c'est le calcul par rapport à la traction de rupture qui donne les résultats les plus défavorables. Les valeurs correspondant à $0.9 T_{C}$ sont légèrement plus optimistes. On vérifie bien que la valeur de $\beta$ décroît quand la longueur du tirant augmente.

\subsection{Déplacements à la charge critique $T_{c}$}

Les valeurs de ces déplacements ont été reportées dans le tableau $n^{\circ} 1$. Le déplacement de la tête du tirant métallique $n^{\circ} 2$, sollicité en traction, a été de $2,5 \mathrm{~mm}$. A titre indicatif, dans le cas de la plaque ce déplacement atteignait $6,5 \mathrm{~mm}$. Le déplacement croît avec la longueur du bulbe, toute chose restant égale par ailleurs. Les valeurs observées pour les tirants $n^{\circ} 4,5$ et 6 de 2,3 et $4 \mathrm{~m}$ de longueur ont été respectivement égales à : $1,5-1,8$ et $2,5 \mathrm{~mm}$.

Les tirants $n^{\circ} 7$ et 8 ont des déplacements identiques $(2,0 \mathrm{~mm})$. Par contre le tirant $n^{\circ} 9$ de même longueur de

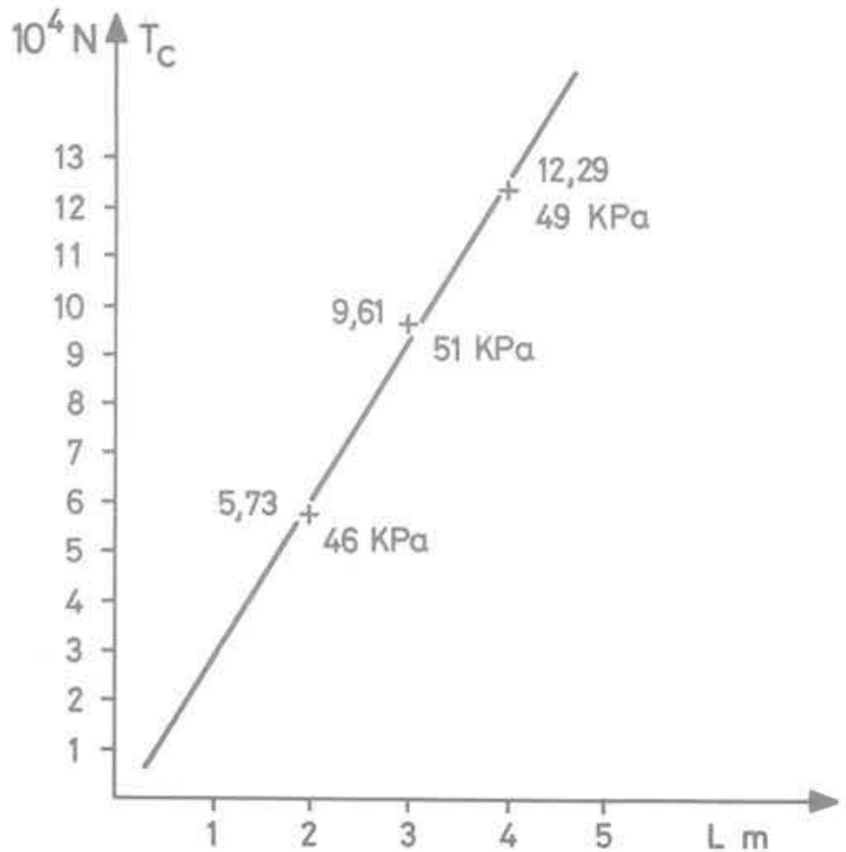

Fig. 7 Evolution de la traction critique $T_{C}$ en fonction de la longueur $L$ du bulbe d'ancrage 


\begin{tabular}{|c|c|c|c|c|c|c|c|c|c|}
\hline $\begin{array}{c}N^{\circ} \\
\text { de l'essai }\end{array}$ & \begin{tabular}{|c|}
\multicolumn{1}{c|}{$\beta$} \\
$\mathrm{mm} / \mathrm{dec} / 10^{4} \mathrm{~N}$ \\
100
\end{tabular} & $\begin{array}{l}T_{c} \\
k N\end{array}$ & $\begin{array}{l}T^{\prime} c \\
k N\end{array}$ & $\begin{array}{l}T_{0} \\
T^{\prime} c\end{array}$ & $\begin{array}{c}T \\
k N\end{array}$ & $\begin{array}{c}\mathrm{T}_{\mathrm{a}}=0,9 \mathrm{~T}_{\mathrm{c}} \\
\mathrm{kN}\end{array}$ & $\begin{array}{c}\mathrm{T}_{\mathrm{a}}=0,8 \mathrm{~T}_{\mathrm{c}} \\
k \mathrm{~N}\end{array}$ & $\begin{array}{c}\mathrm{T}_{\mathrm{a}}=\mathrm{T} \\
\mathrm{kN} \quad 1,5\end{array}$ & $\begin{array}{c}\text { déplacement } \\
\text { à } T_{c} \\
\text { mm }\end{array}$ \\
\hline 2 & 1,42 & 49,2 & 54,1 & 0,91 & 68,9 & 36,8 & 43,3 & 45,9 & 2,5 \\
\hline 3 & 2,51 & 56,4 (1) & 62,0 & 0,91 & 73.3 & 50,8 & 49,6 & 48,9 & \\
\hline 4 & 2,22 & 57,3 & 65,5 & 0,87 & 76,4 & 51,6 & 52,4 & 50,9 & $1,5(3)$ \\
\hline 5 & 1,37 & 96,1 & 105,9 & 0,91 & 120,1 & 86,5 & 84.7 & 80,1 & $1,8(3)$ \\
\hline 6 & 1,08 & 122,9 & 135,4 & 0,91 & 150,1 & 110,6 & 108,3 & 100,1 & $2,5(3)$ \\
\hline 7 & 1,55 & 90.1 & 104.7 & 0,86 & $106,5(2)$ & 81.1 & 83,8 & 71,0 & $2,0(3)$ \\
\hline 8 & 1,61 & 95,6 & 107,0 & 0.89 & 122,9 & 86,0 & 85,6 & 81,9 & $2,0(3)$ \\
\hline 9 & 1.54 & 65,5 & 75,9 & 0,86 & 86.3 & 59,0 & 60,7 & 57,5 & $1,5(3)$ \\
\hline
\end{tabular}

(1) Fluage en pied du bulbe

(2) Valeur des incréments de charge trop élevée

(3) Valeurs mesurées après fluage

Tableau 1

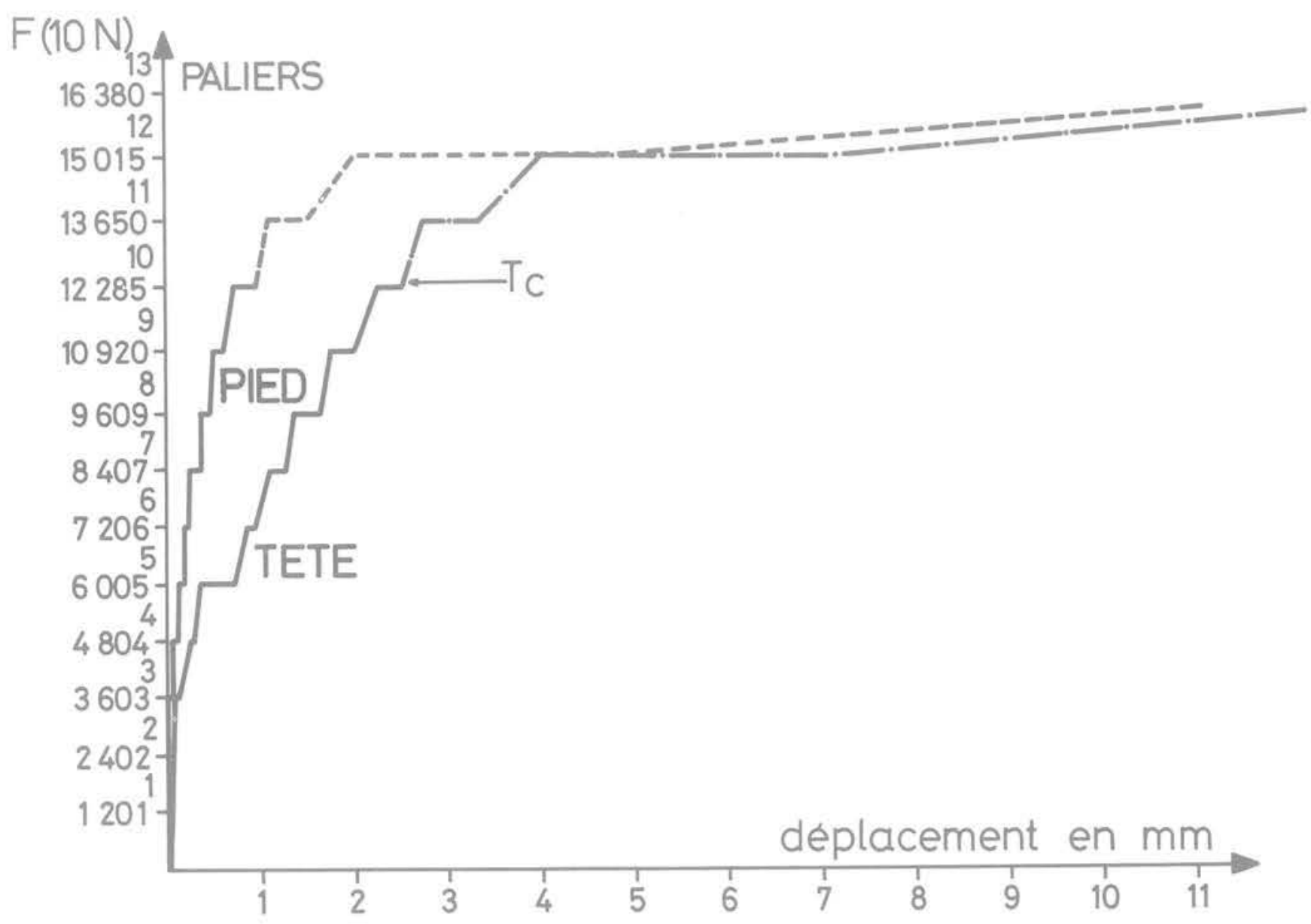

Fig. 8 Déplacement en tête et en pied du tirant $n^{\circ} 6$ au cours des paliers de chargement

bulbe et placé sous la mẻme hauteur de sable que les précédents mais dont la butée frontale a été supprimée à une déplacement plus faible $(1,5 \mathrm{~mm})$ duquel il faut rapprocher une valeur de $T_{C}$ plus faible également.

Sur la figure n 8 ont été reportés les déplacements en tête et en pied du tirant $n^{\circ} 6$, en fonction des charges appliquées à ce tirant. On peut constater que le déplacement du pied se produit dès le troisième palier. On peut également noter un fluage, au niveau de la tête, anormalement important dans le cinquième palier à cause d'une fissuration importante du coulis au cours de ce palier.

Pour la traction critique $T_{c}$ les valeurs des déplacements en tête et en pied, en fin de fluage, sont respectivement de 2,5 et $1,0 \mathrm{~mm}$. 


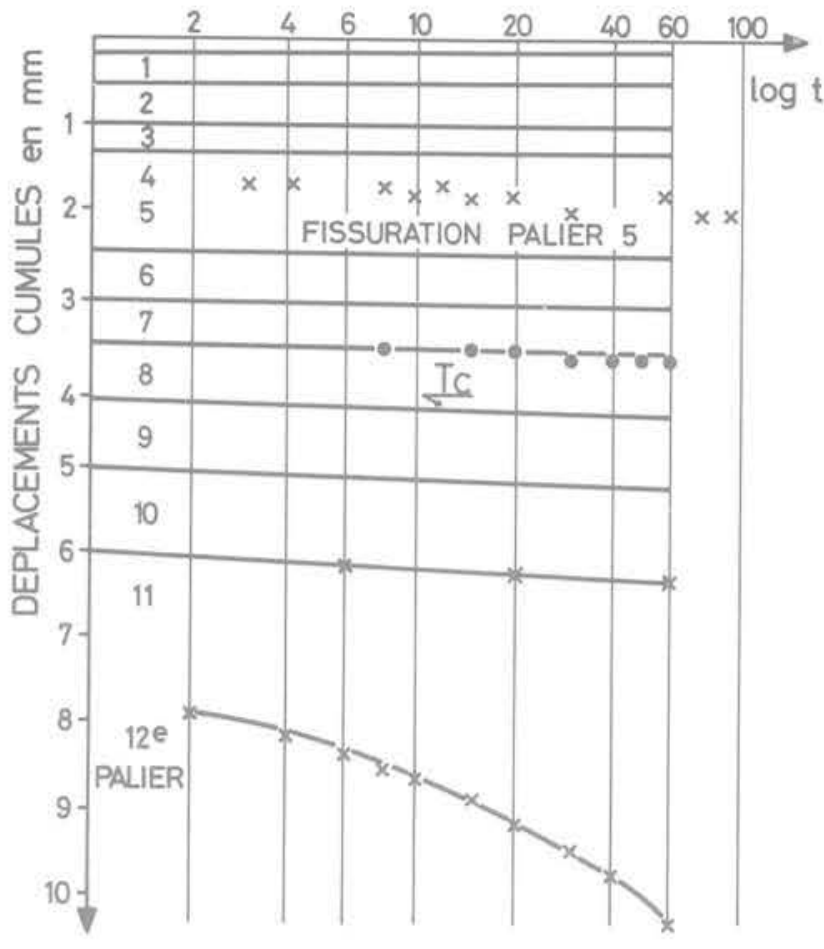

Fig. 9 Courbes de fluage du tirant $n^{\circ} 6$

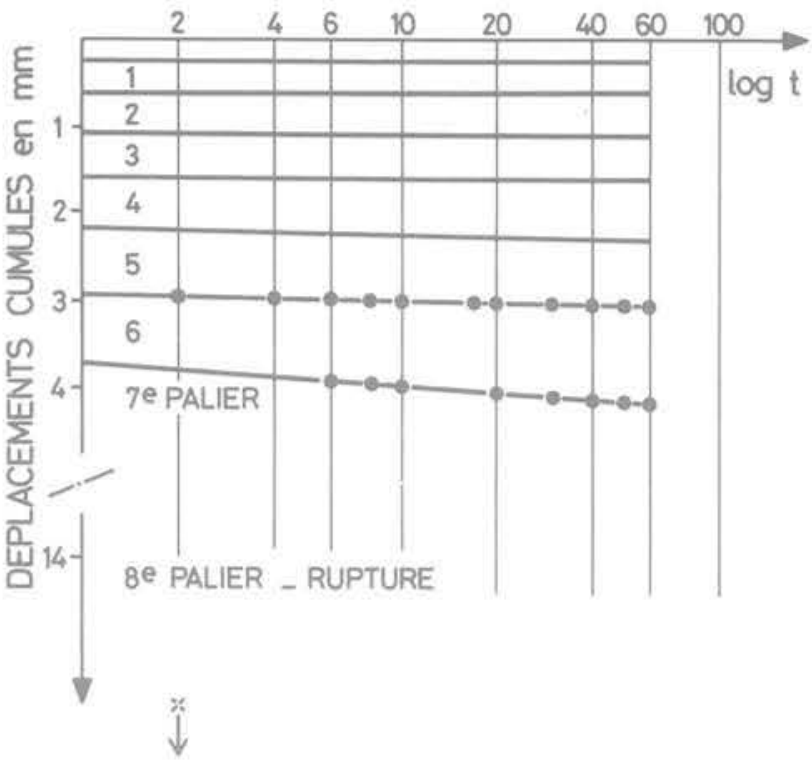

Fig. 10 Courbes de fluage du tirant $n^{\circ} \mathrm{g}$

\subsection{Courbes de fluage}

Les courbes de fluage de tous les tirants sont linéaires jusqu'à la traction critique.

La figure 9 donne, à titre d'exemple, les courbes de fluage du tirant $n^{\circ} 6$. On peut constater qu'elles restent linéaires jusqu'au onzième palier (la traction critique a été atteinte au dixième palier).

A cause de la fissuration survenue au cours du cinquième palier il n'a pas été possible de tracer la courbe correspondant à ce palier. Le douzième palier possède une courbure assez forte. Le treizième palier n'a pas été représenté.

Il est à noter que la rupture franche n'a jamais été obtenue à l'exception du tirant $n^{\circ} 9$ dont la butée frontale avait été supprimée. Les courbes de fluage correspondant à ce tirant sont représentées sur la figure 10 . Ces courbes étaient linéaires jusqu'au septième palier. Au huitième palier le tirant a cédé brusquement sans qu'il soit possible de tracer une courbe de fluage.

\subsection{Pression transmise au milieu}

Sur la figure $n^{\circ} 11$ sont représentées, en fonction des tractions appliquées au tirant $n^{\circ} 6$, les pressions enregistrées par les capteurs situés devant le bulbe de ce tirant. A la traction critique $T_{C}$ ces valeurs sont faibles ( 80 à $200 \mathrm{k} \mathrm{Pa}$ ). ('). Les pressions enregistrées par les capteurs situés latéralement au bulbe (capteur 205 et 206) ont augmenté nettement (fig. 12). Les capteurs situés au-dessus du tirant (capteur $221-200$ et 204) n'ont enregistré que des pressions faibles ou même décroissantes.

Des résultats de mème nature ont été obtenus dans tous les essais. La figure 13 représente les résultats correspondant au tirant $n^{\circ} 9$. On constate bien globalement les mêmes résultats que ceux qui viennent d'être décrits. Les pressions

(1) Pour des tractions supérieures à T la pression croìt à une allure exponentielle.

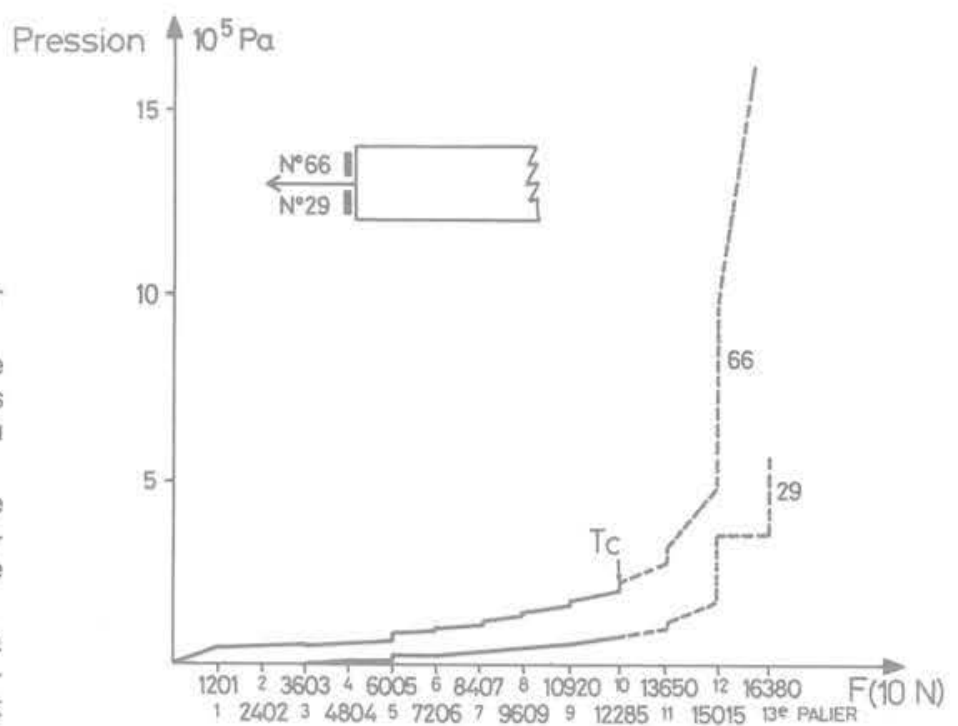

Fig. 11 Evolution, en fonction des tractions appliquées au tirant $n^{\circ} 6$, des pressions devant le bulbe

transmises aux capteurs latéraux (203 et 300 ) croissent nettement et ateignent des valeurs de l'ordre de $25 \mathrm{k} \mathrm{Pa}$ à la traction critique. Au-dessous de la traction critique les capteurs situés au-dessus du bulbe (200-202 et 198) donnent des accroissements de pression faibles, à l'exception du capteur 216 qui accuse des pressions plus importantes. Le capteur 204 donne des accroissements de pression de l'ordre de celles des capteurs latéraux. Le capteur 221 situé audessous du bulbe mais à une distance plus importante que celle du capteur 204 a, de ce fait, enregistré des pressions nettement plus faibles. 


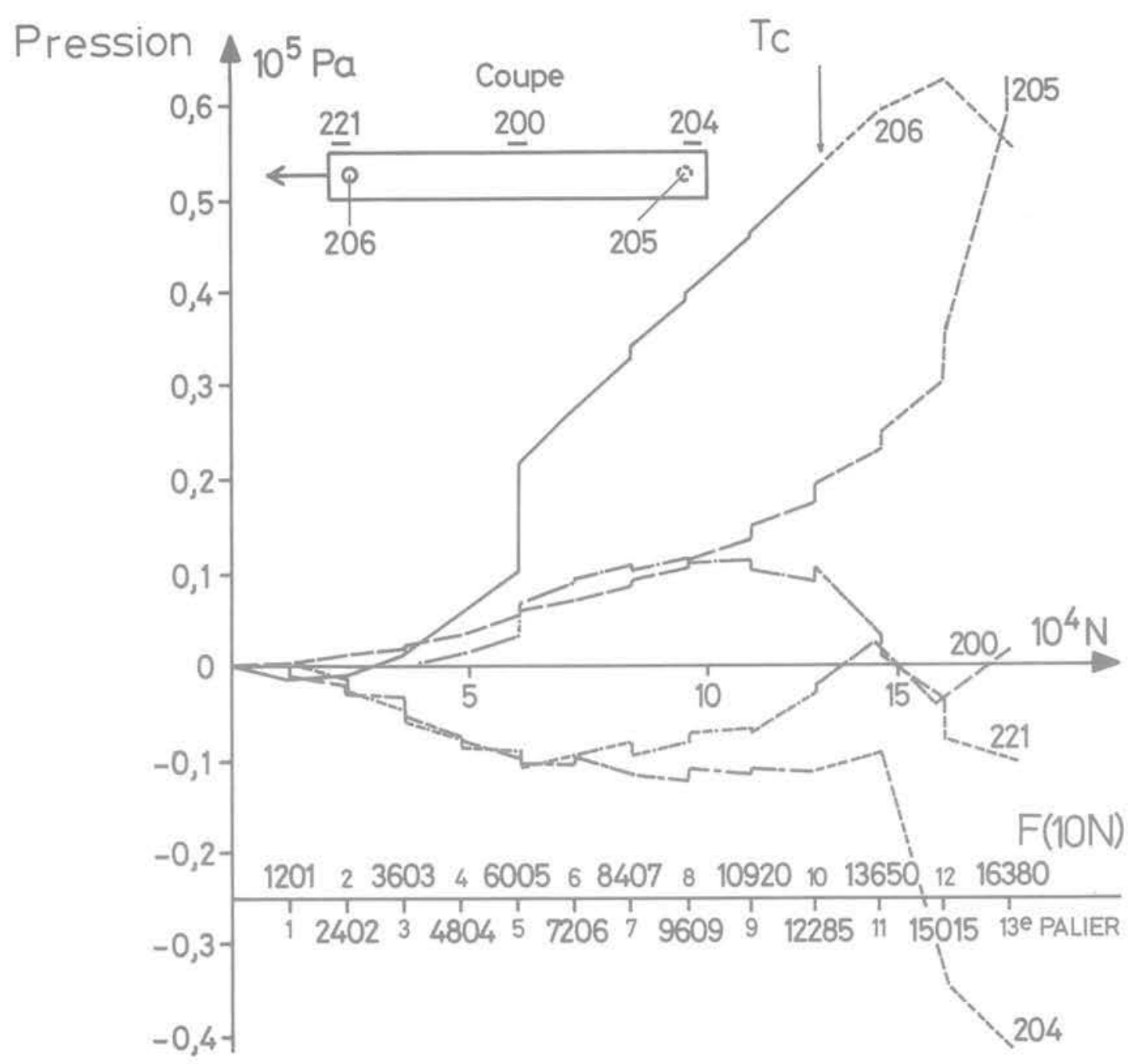

Fig. 12 Evolution des pressions latérales en fonction des tractions appliquées au tirant $n^{\circ} 6$

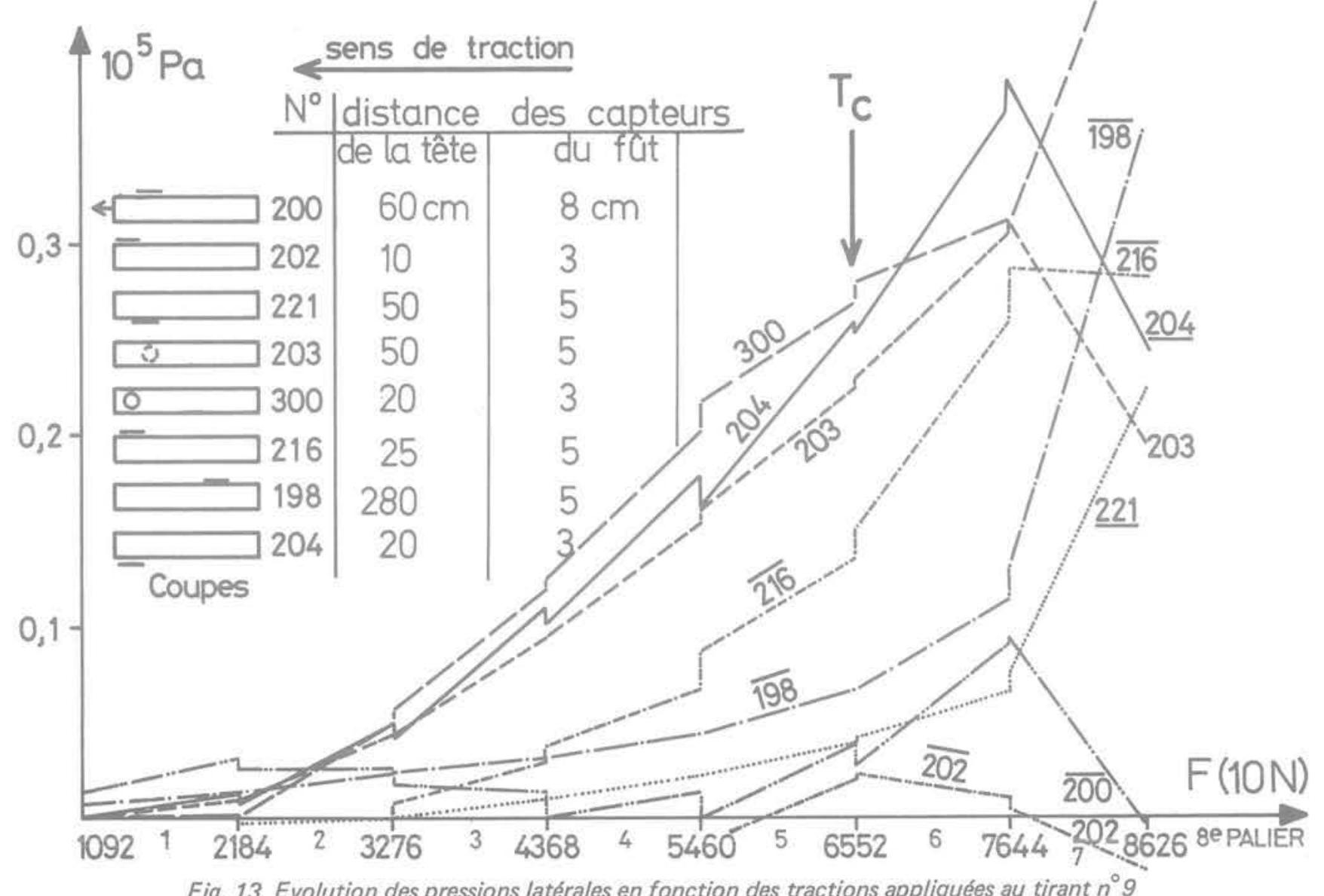

Fig. 13 Evolution des pressions latérales en fonction des tractions appliquées au tirant $n^{\circ} \mathrm{g}$ 


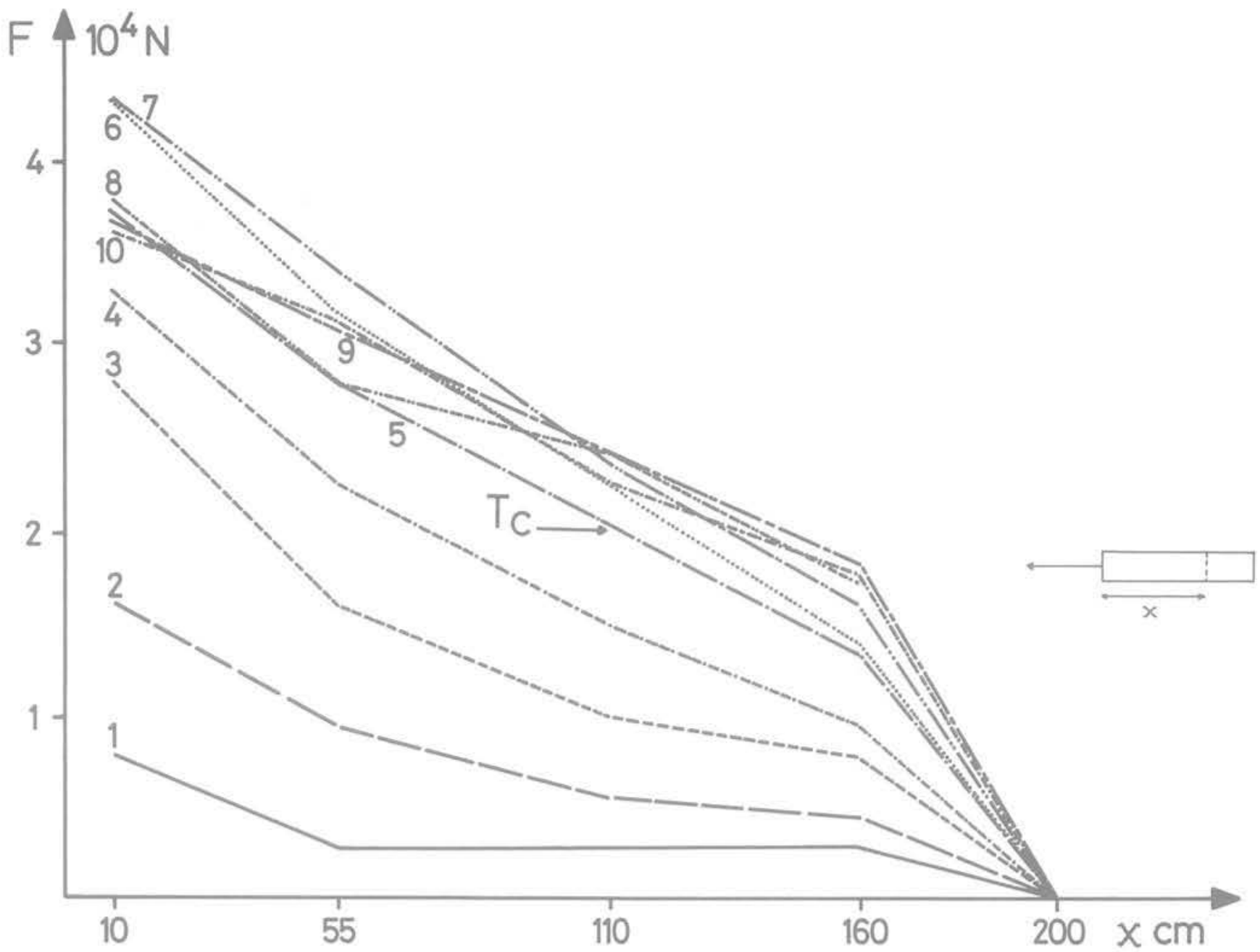

Fig. 14 Evolution de l'effort total le long du bulbe du tirant $n^{\circ} 2$

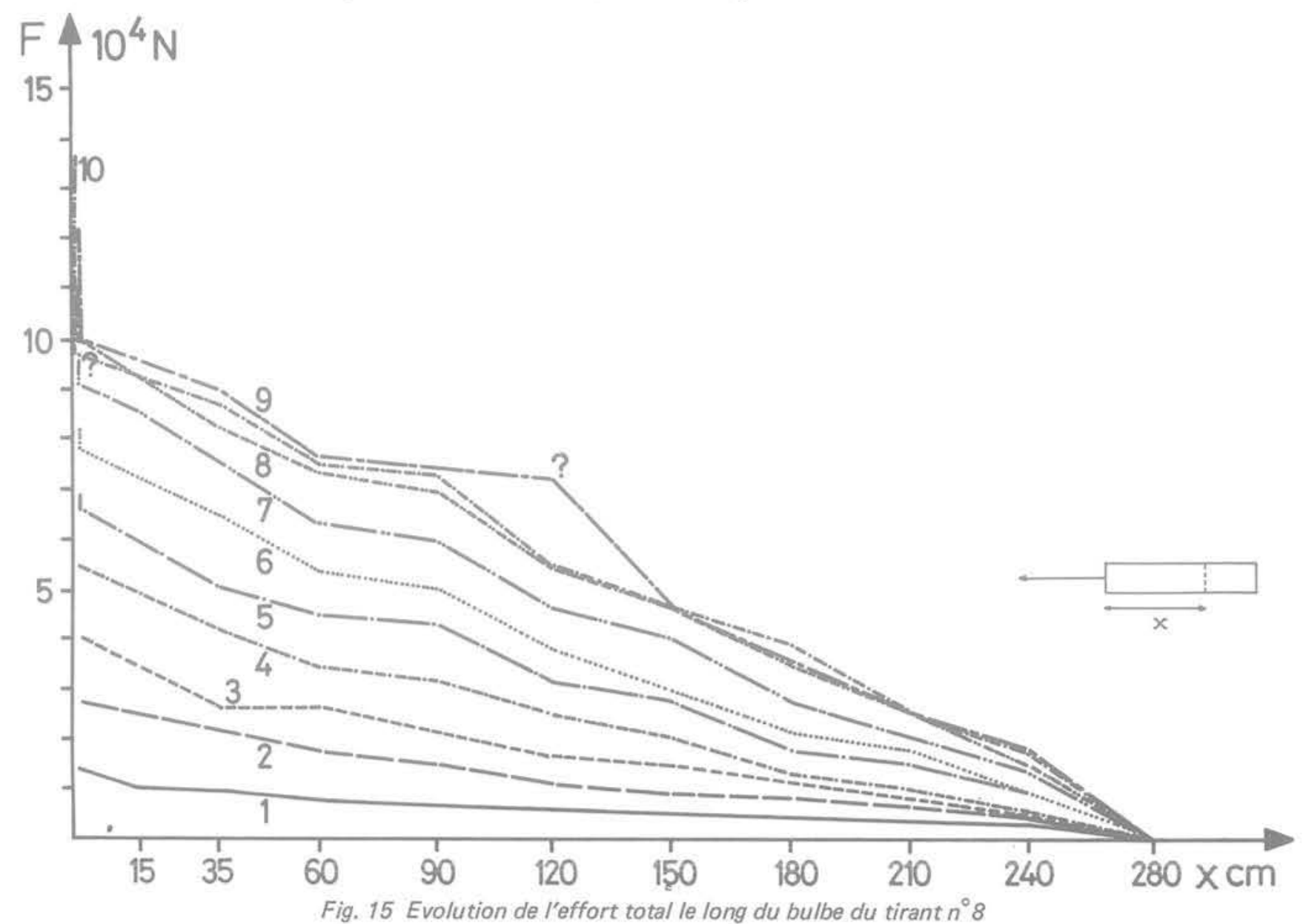




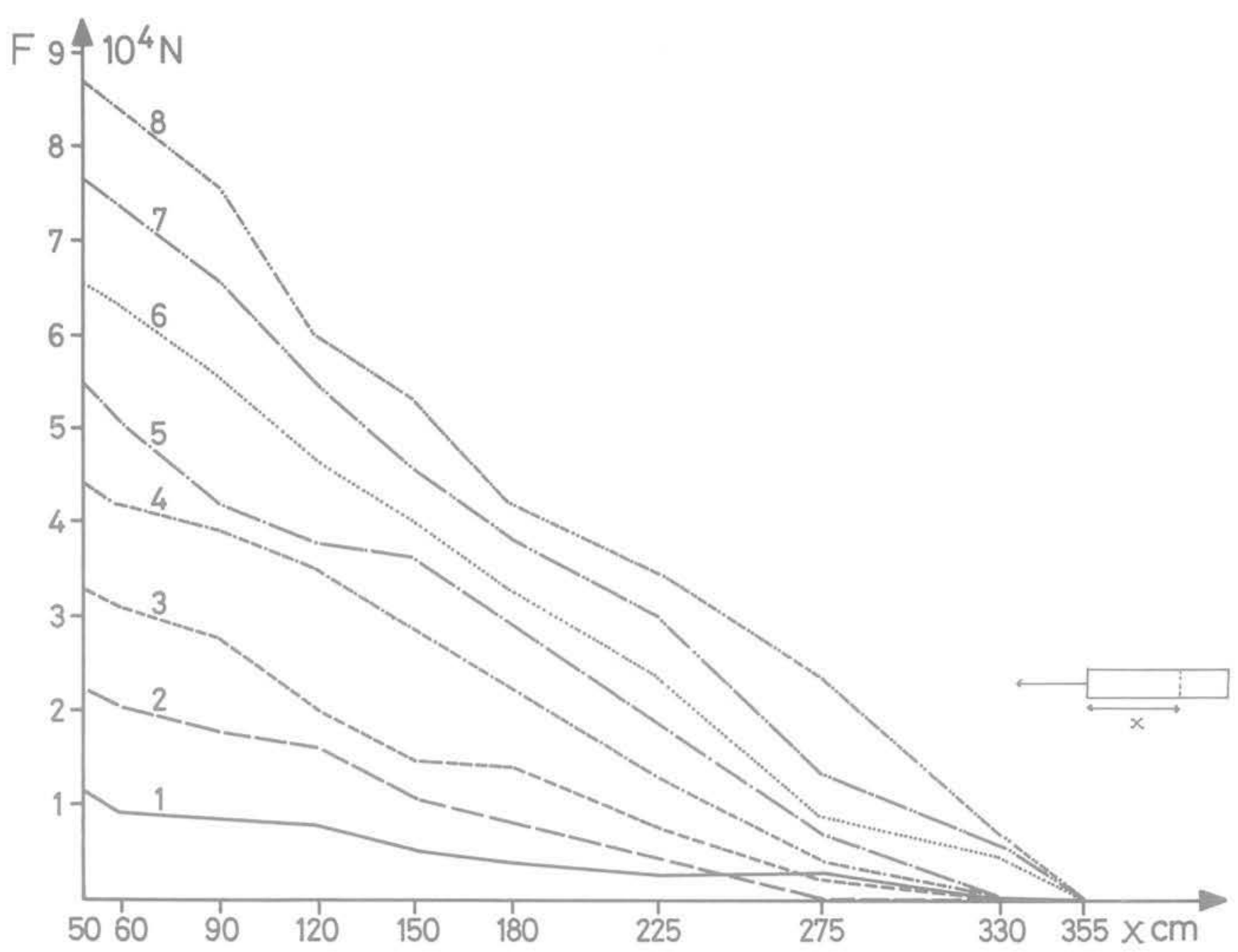

Fig. 16 Evolution de l'effort total le long du bulbe du tirant $n^{\circ} 9$

\subsection{Répartition des efforts le long du bulbe du tirant}

Les jauges de déformation placées sur les demi tubes des tirants métalliques ou sur l'armature des tirants en coulis ont permis de déterminer la répartition des efforts le long du bulbe. Par contre les mesures des jauges noyées dans le coulis n'ont pu être exploitées par suite d'un défaut de connexion.

L'étalonnage avant essai des jauges des tirants en coulis a été complété par un nouvel étalonnage après essai afin de tenir compte des effets de la fissuration des coulis.

La figure 14 donne l'évolution de l'effort le long du tirant métallique $n^{\circ} 2$. La traction critique $T_{c}$ était atteinte au cinquième palier. On peut constater qu'à partir du septième palier les courbes deviennent très voisines ce qui montre que les accroissements de charges sont alors entièrement absorbés par l'accroissement de la butée frontale.

Des phénomènes semblables peuvent être observés dans le cas du tirant en coulis $n^{\circ} 8$ (fig. 15) dont la traction critique est atteinte au septième palier. A partir du huitième palier les courbes deviennent très voisines et l'accroissement des efforts appliqués au tirant est absorbé par la mise en butée progressive de la tête du bulbe.

Dans le cas du tirant $n^{\circ} 9$, pour lequel la butée frontale a été supprimée, la répartition des efforts évolue régulièrement jusqu'à la rupture (fig. 16). Pour l'ensemble des essais on a pu constater que dès les premiers paliers de chargement la partie arrière du bulbe était sollicitée par des efforts latéraux. D'autre part l'effort latéral total augmente encore pour le palier succédant à la traction critique puis chute ensuite légèrement.

\subsection{Frottement latéral unitaire $\tau_{m}$}

Les valeurs du frottement latéral unitaire ont été déterminées pour des longueurs d'environ $60 \mathrm{~cm}$. La fissuration des coulis a parfois entraîné des difficultés de mesure et par suite une certaine imprécision dans la détermination des valeurs du frottement unitaire.

La figure 17 représente la distribution de $\tau_{m}$ le long du bulbe, pour le tirant métallique $n^{\circ} 2$. On peut constater que pendant les quatre premiers paliers la valeur maximale de $\tau_{m}$ est située à la tête du bulbe. Pour les paliers suivants ce maximum passe à l'extrémité arrière du bulbe. Les valeurs de $\tau_{m}$ à cette extrêmité sont très élevées dés le départ, à cause d'une assez grande rigidité du bulbe métallique.

La figure $n^{\circ} 18$ montre nettement, pour le tirant $n^{\circ} 6$, le déplacement de l'avant vers l'arriêre de $\tau$ m maximum quand les charges augmentent. Cette valeur maximale se situe en tête du bulbe pour les cing premiers paliers, au milieu pour les paliers 6 à 9 et vers l'arrière pour les paliers 10 à 13 .

Dans le cas du tirant $n^{\circ} 8$ (fig. 19) quelques valeurs aberrantes ont été observées au cours du neuvième palier. Ces valeurs aberrantes étant mise à part, on n'observe plus de pic dans les courbes de répartition de l'effort latéral unitaire. Les valeurs maximales de cet effort restent en tête pendant la totalité du chargement. On observe une montée progressive des efforts unitaires le long du bulbe qui conduit, à la fin de l'essai, à une valeur relativement constante tout le long du bulbe.

Dans le cas du tirant $n^{\circ} 9$ dépourvu de butée frontale, la distribution du frottement latéral unitaire tend également vers une certaine uniformisation avec l'accroissement des charges (fig. 20). 


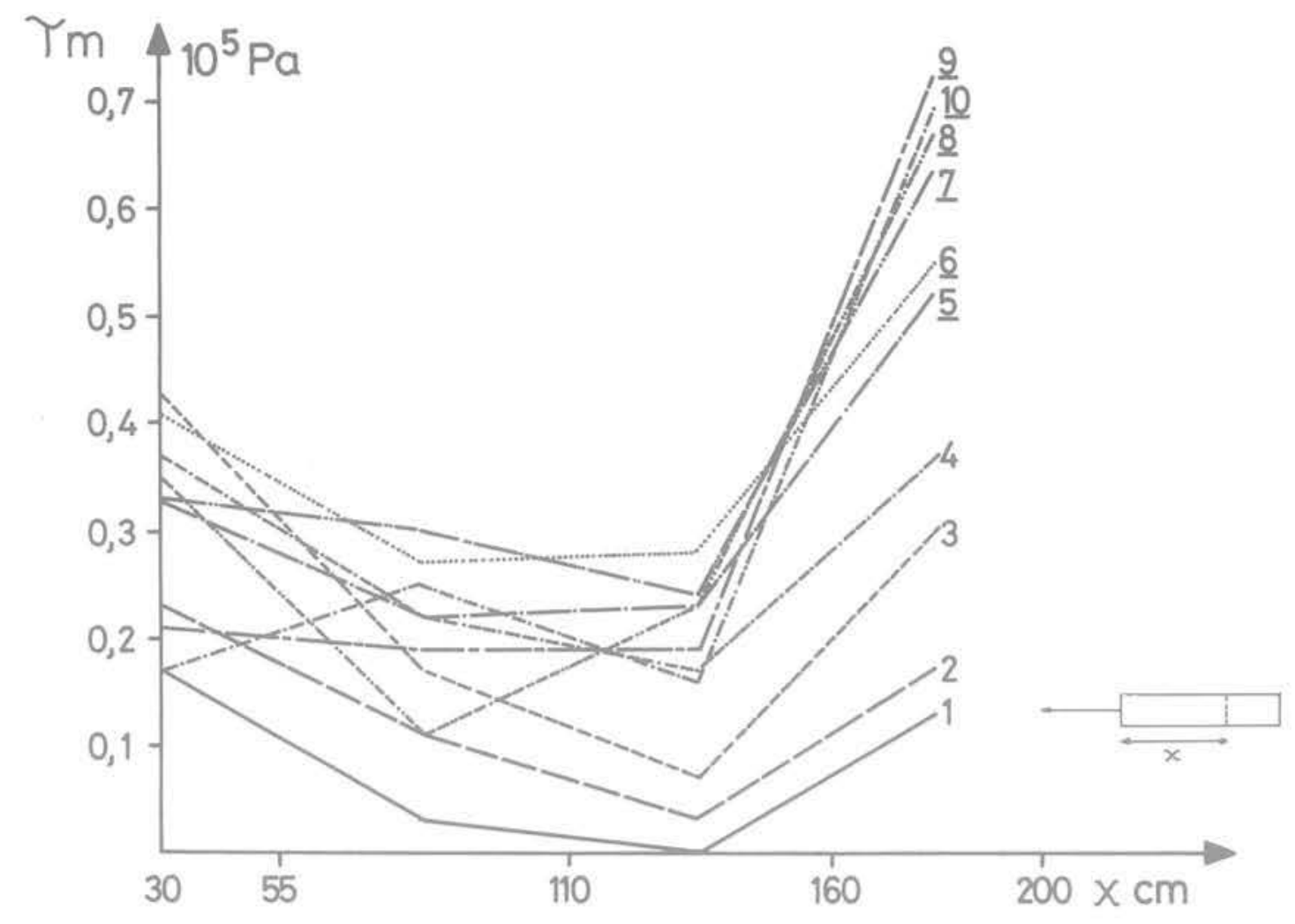

Fig. 17 Evolution du frottement latéral unitaire $\mathrm{T}_{m}$ le long du bulbe du tirant $n^{\circ} 2$



Fig. 18 Evolution du frottement latéral unitaire $T_{m}$ le long du bulbe du tirant $n^{\circ} 6$ 


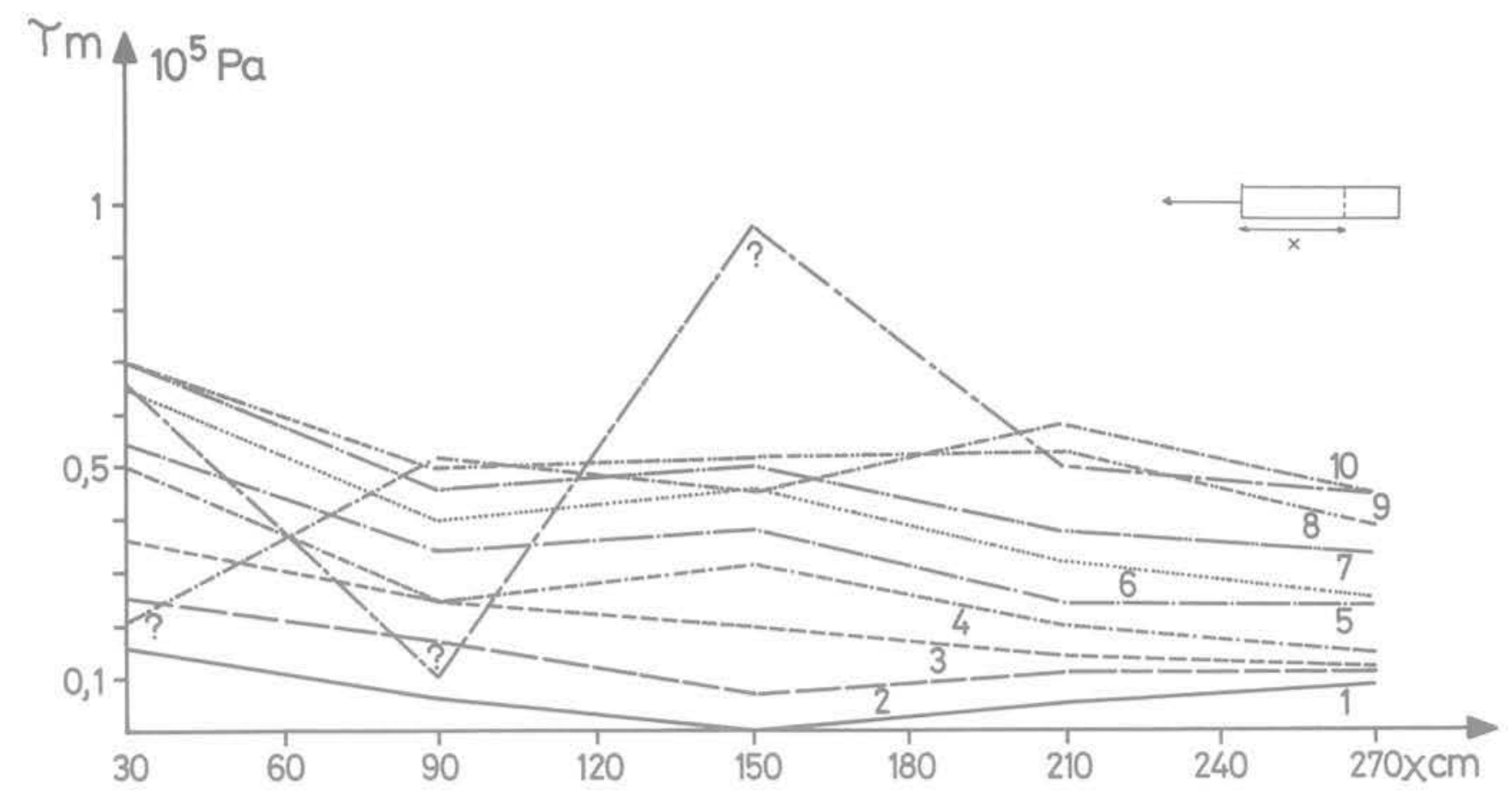

Fig. 19 Evolution du frottement latéral unitaire $T_{m}$ le long du bulbe du tirant $n^{\circ} 8$

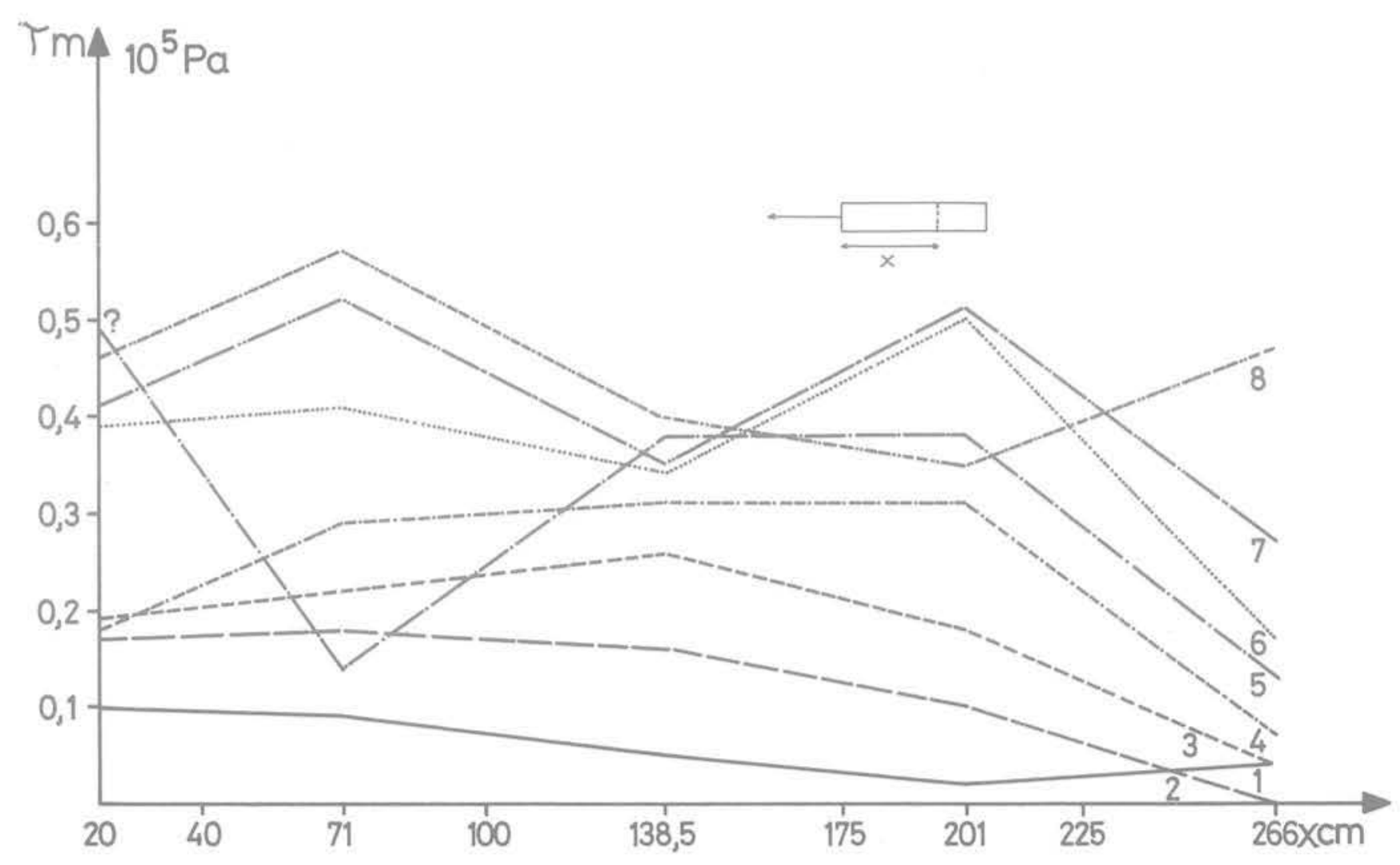

Fig. 20 Evolution du frottement latéral unitaire $\tau_{m}$ le long du bulbe du tirant $n^{\circ} 9$ 


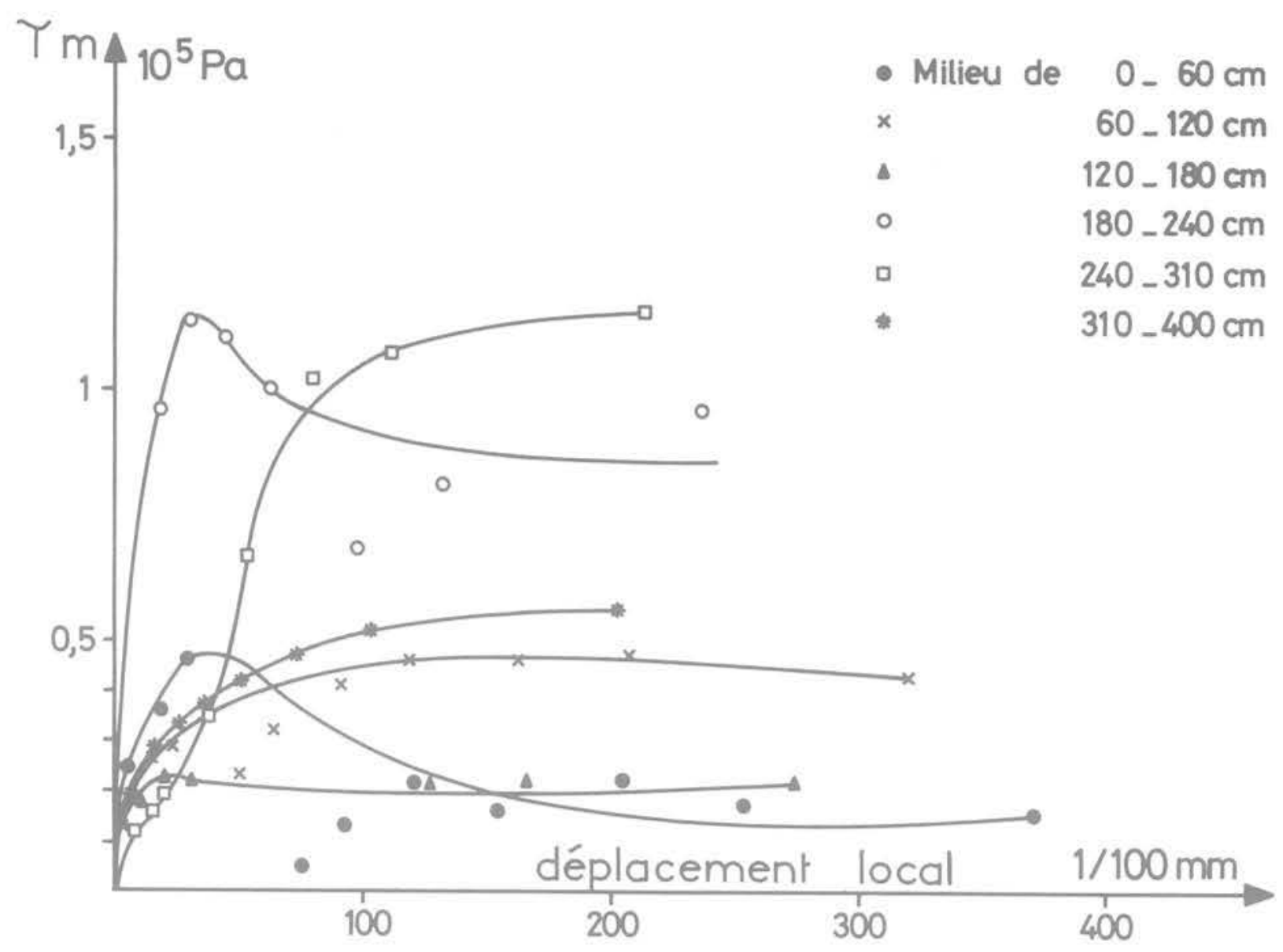

Fig. 21 Variation du frottement latêral unitaire en fonction du déplacement local pour le tirant $n^{\circ} 6$

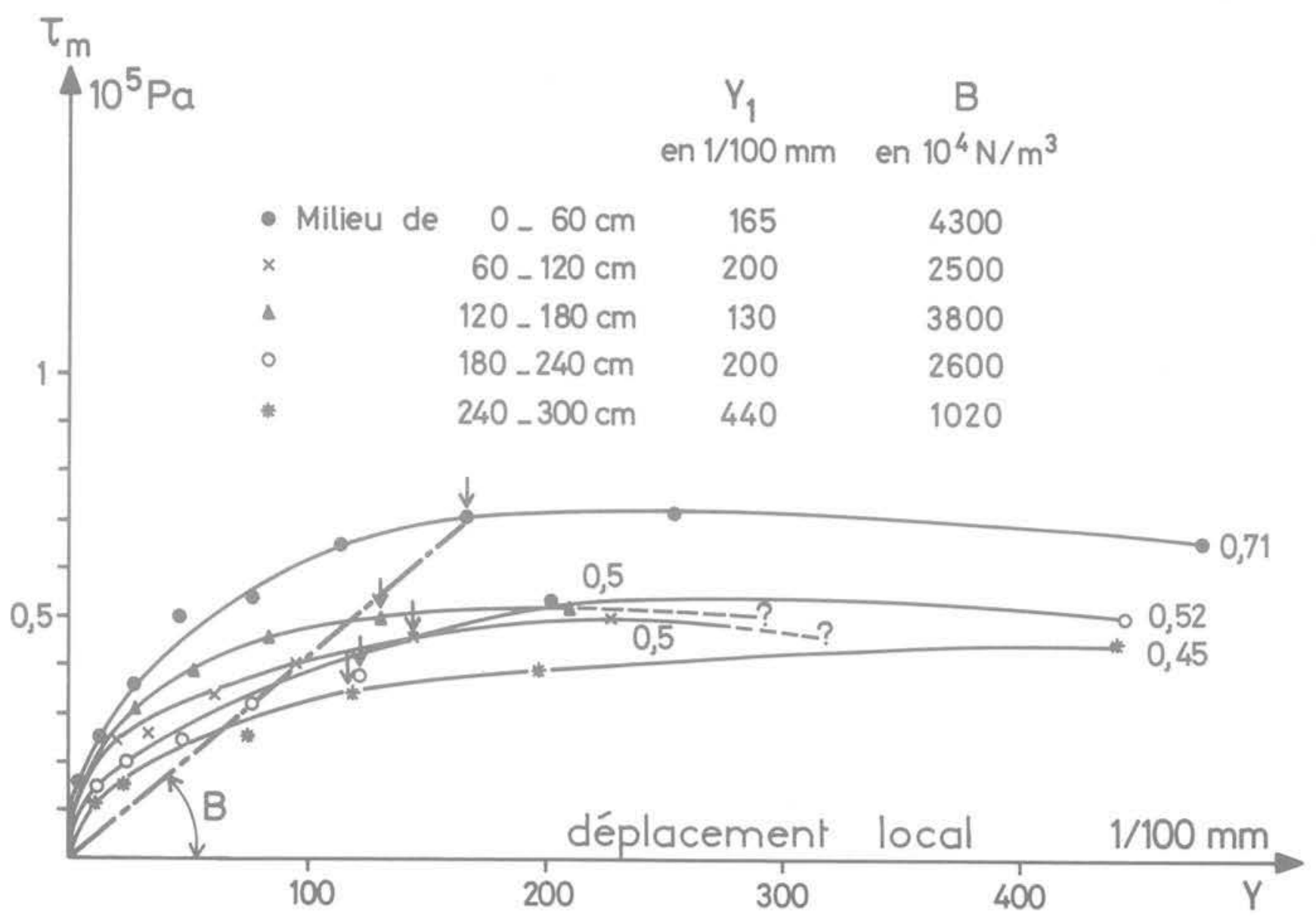

Fig. 22 Variation du frottement latéral unitaire en fonction du déplacement local pour le tirant $n^{\circ} 8$ 


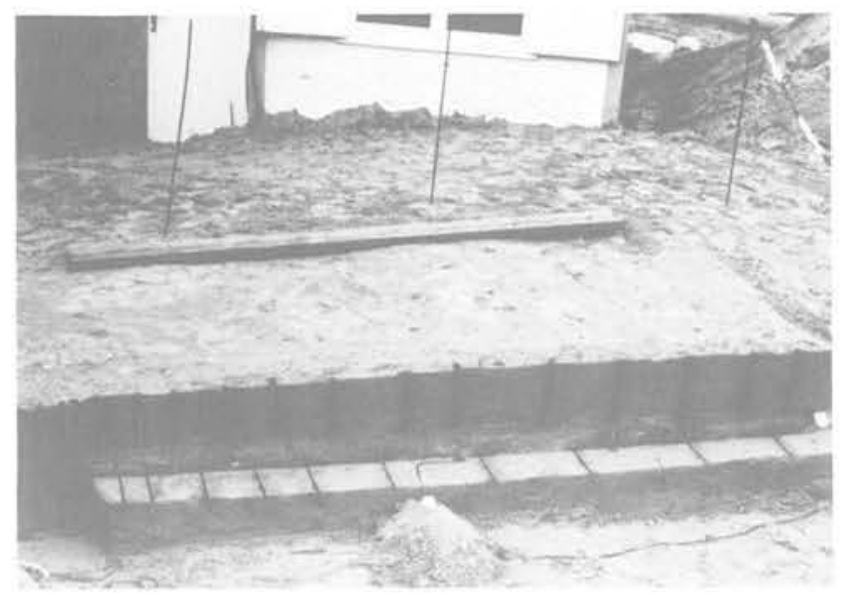

Fig. 23 Vue d'ensemble des bandes et des tubes en sable noir

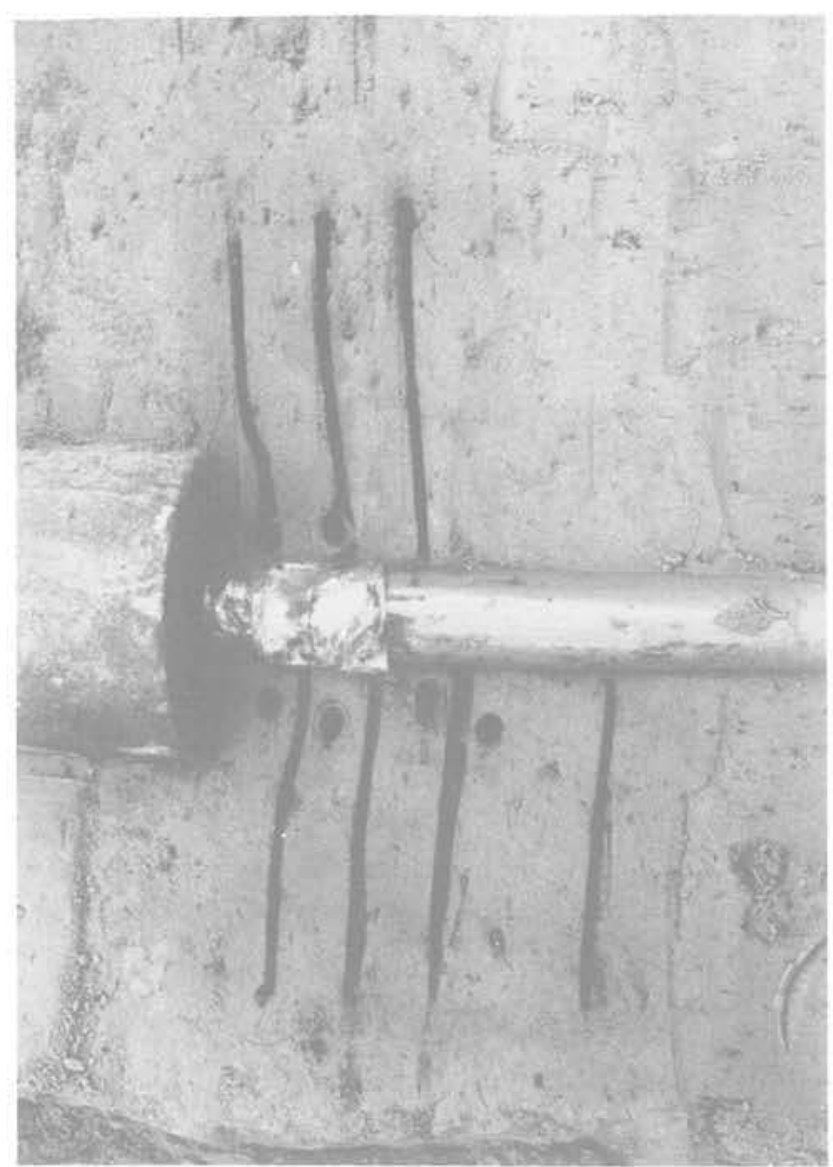

Fig. 24 Exemple de déformations des bandes en sable noir placées devant la tête du bulbe

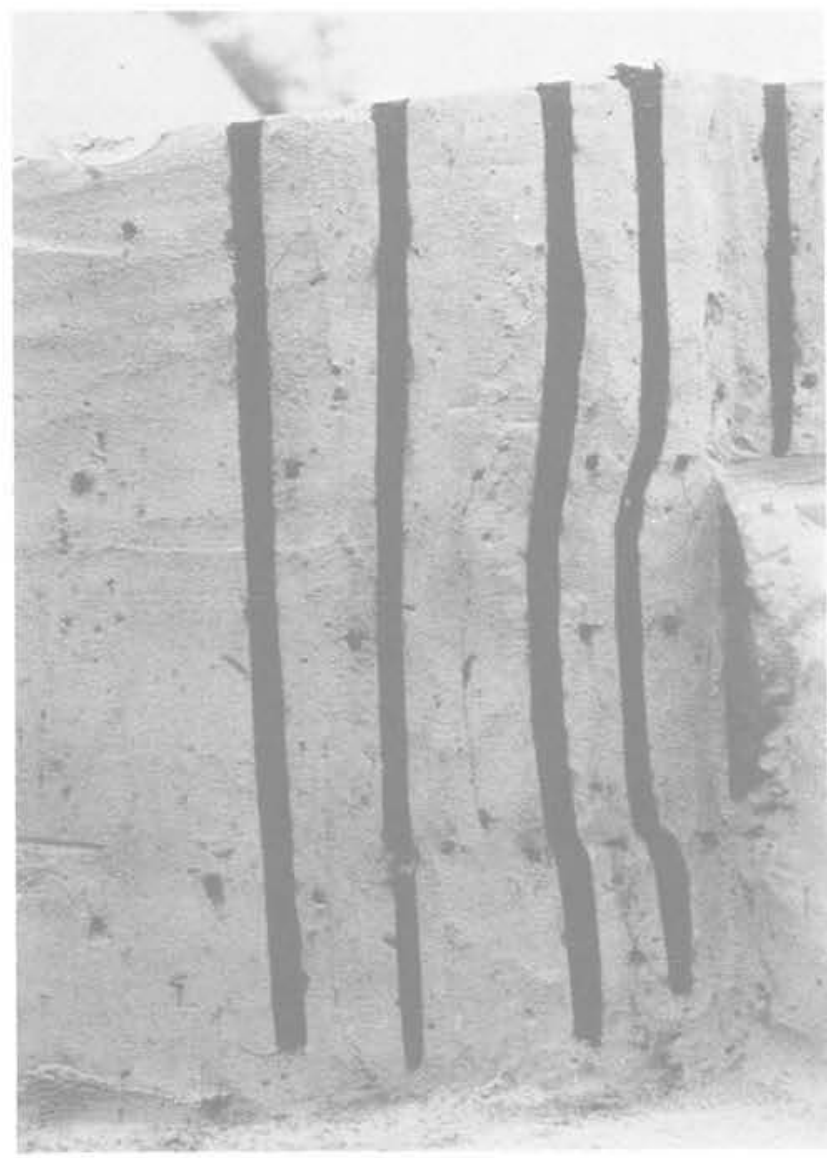

Fig. 25 Exemple de déformations des tubes en sable noir placés devant la tête du bulbe

\subsection{Variations du frottement latéral unitaire en fonction du déplacement local}

La figure 21 donne les courbes correspondant au tirant $n^{\circ} 6$. On peut constater une certaine dispersion dans les valeurs mesurées. Deux des courbes présentent un pic et les autres un accroissement régulier, $P$ ar contre le tirant $n^{\circ} 8$ (fig. 22), les courbes présentent toutes des paliers compris entre 40 $\mathrm{kPa}$ et $70 \mathrm{kPa}$, pour des déplacements locaux compris entre 1,6 et $2,0 \mathrm{~mm}$.

Sur cette dernière figure on a également indiqué les paramètres $Y_{1}$ et $B$ définis par Cambefort [2]. Le point $Y_{1}$ correspond au déplacement du coulis par rapport au sable pour lequel le frottement latéral est saturé. La valeur de $B$ a été prise égale à la pente de la droite joignant le point $Y_{1}$ à l'origine.

\subsection{Déformations du milieu sableux}

Les déformations du milieu sableux à la fin de l'essai ont été obtenues par l'observation des bandes et des tubes en sable noir. La figure 23 donne une vue d'ensemble des bandes et des tubes en sable noir placés autour du bulbe et des tubes en sable noir placés devant la tête du tirant. Dans l'exemple de bandes et de tubes en sable noir placés devant la tête du bulbe, donné par les figures 24 et 25 , la zone déformée était de faible dimension malgré un déplacement de la tête du bulbe de l'ordre de $20 \mathrm{~mm}$.

Cette zone déformée avait, en moyenne, une longueur de l'ordre de $17 \mathrm{~cm}$, une largeur de l'ordre de $35 \mathrm{~cm}$ et une hauteur de l'ordre de $28 \mathrm{~cm}$.

L'examen des tubes et des bandes en sable noir placés au voisinage du bulbe a montré que la zone cisaillée n'avait qu'une très faible épaisseur, de l'ordre de 1 à $2 \mathrm{~mm}$ (fig. 26 e 27). On peut en particulier observer sur la figure 27 la traînée de sable noir causée par le déplacement du tirant. 

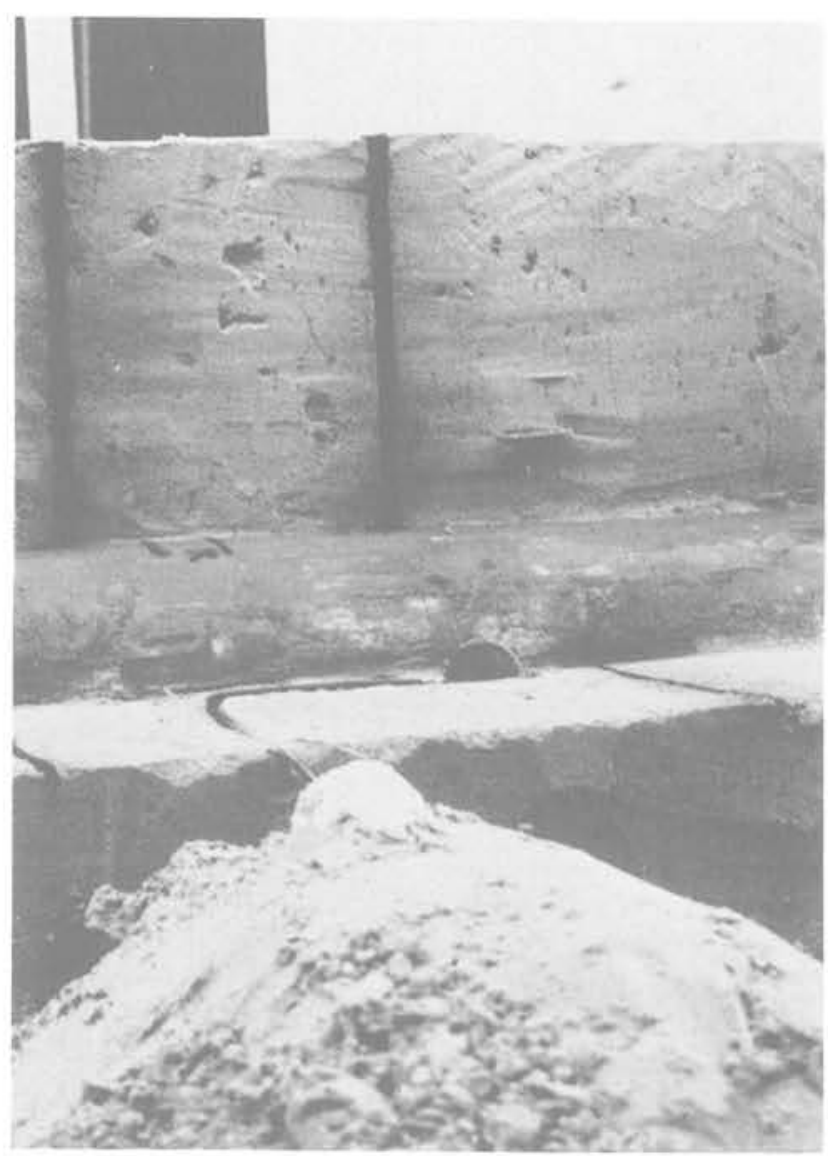

Fig. 26 Exemple de déformations des tubes en sable noir placés au-dessus du bulbe

\section{Conclusions}

Cette première série d'essais a apporté une meilleure compréhension du comportement des tirants d'ancrage. Les couches de répartition des efforts le long du bulbe et d'évolution des efforts devant la tête de l'ancrage ont permis de mettre en évidence, qu'après mobilisation totale de l'effort latéral peu après la traction critique $T_{c}$, les efforts supplémentaires appliqués au tirant étaient absorbés intégralement par la butée en tête du bulbe. Cette reprise des efforts s'accompagne de vitesses de fluage importantes.

Le critère de rupture est difficile à définir. La traction de rupture pourrait être définie par la valeur correspondant è un palier de chargement pour lequel la pente descourbes de stabilisation serait supérieure à $1 \mathrm{~mm}$ par décade de minutes.

Les essais d'ancrages en coulis de $3 \mathrm{~m}$ de longueur de bulbe et ancrés sous des profondeurs de sable de 2 et $3 \mathrm{~m}$ ont montré que la profondeur critique, pour laquelle les efforts de traction sont indépendants de la profondeur d'ancrage, est inférieure à $2 \mathrm{~m}$.

La traction critique augmente linéairement en fonction des longueurs d'ancrage pour des valeurs comprises entre 2 et 4 $\mathrm{m}$. Ces résultats, obtenus dans un milieu constitué par un sable fin de granulométrie uniforme et un indice de densité égal 0,59 confirment les résultats de Ostermayer [4]

Les témoins en sable noir ont montré que la zone cisaillée autour du bulbe n'avait qu'une épaisseur très faible de l'ordre de 1 à $2 \mathrm{~mm}$. La zone de rupture devant la tête de bulbe n'a également que des dimensions très limitées.

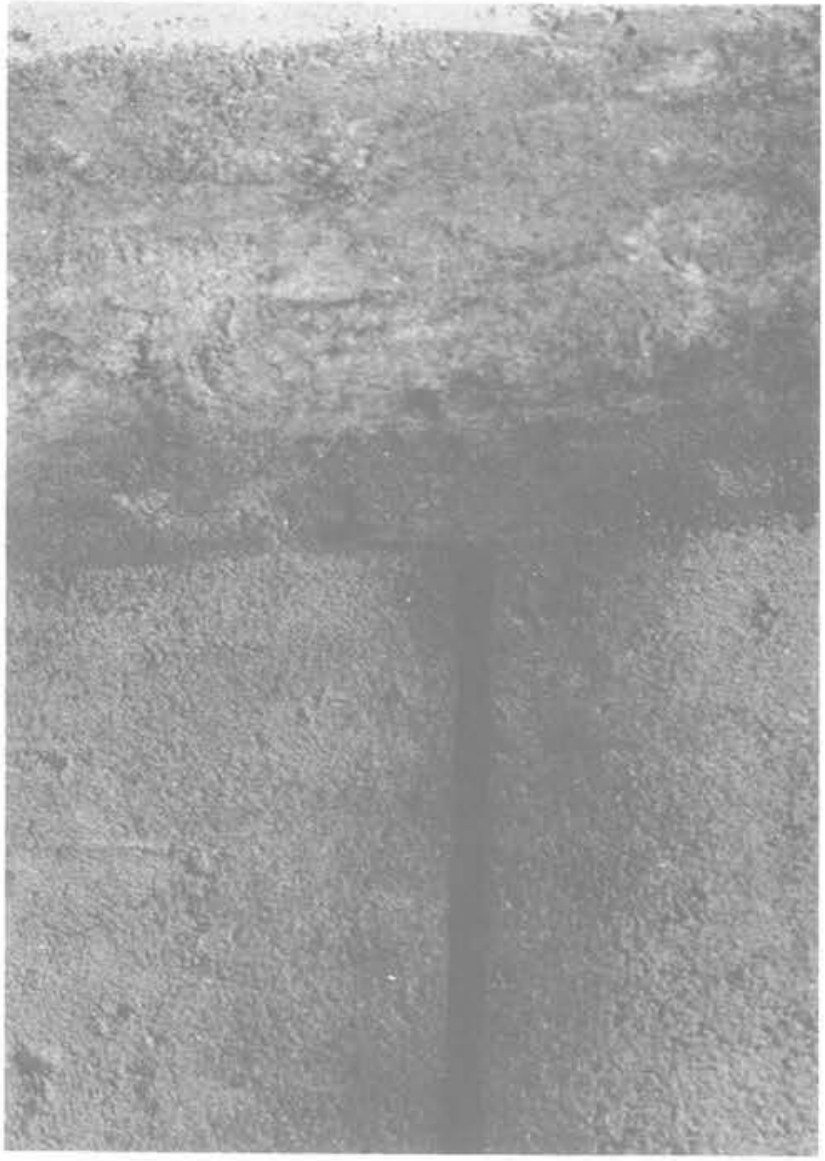

Fig. 27 Exemple de déformations d'une bande de sable noir placée contre le bulbe

Des essais de tirants possédant des bulbes de plus grande longueur seront réalisés dans une phase ultérieure. Ils doivent permettre d'affiner les connaisances sur l'évolution du frottement latéral en fonction des charges et sur le déplacement du bulbe par rapport au sable et d'adapter aux tirants d'ancrage les lois proposées par Cambefort [2] pour les pieux.

\section{Références bibliographiques}

[1] BUSTAMANTE M., DELMAS F., LACOUR J. (1978) "Comportement des tirants précontraints dans une argile plastique. Revue Française de Géotechnique n ${ }^{\circ} 3$ - Janvier 1978.

[2] CAMBEFORT, CHADEISSON (1965) - "Critère pour l'évaluation de la force portante d'un pieu C $R$. $6^{\circ}$ Congrès International de Mécanique des Sols et Fondations. Montréal (1965).

[3] LOGEAIS L., BUSTAMANTE M. "Les tirants d'ancrage". La nouvelle édition des Recommandations. Annales de III.T.B.T.P. n 356 - Décembre 1977.

[4] OSTERMAYER H., SCHEELE F. (1978) - "Research in ground anchors in non cohesive soils." Revue Française de Géotechnique n³ - Janvier 1978. 
